TRANSACTIONS OF THE

AMERICAN MATHEMATICAL SOCIETY

Volume 357, Number 5, Pages 1731-1751

S 0002-9947(04)03714-6

Article electronically published on December 22, 2004

\title{
COISOTROPIC AND POLAR ACTIONS ON COMPLEX GRASSMANNIANS
}

\author{
LEONARDO BILIOTTI AND ANNA GORI
}

\begin{abstract}
The main result of the paper is the complete classification of the compact connected Lie groups acting coisotropically on complex Grassmannians. This is used to determine the polar actions on the same manifolds.
\end{abstract}

\section{INTRODUCTION AND PRELIMINARIES}

The aim of this paper is to present the classification of compact connected subgroups of $\mathrm{SU}(n)$ acting coisotropically on the complex Grassmannians $\operatorname{Gr}(k, n)=$ $\mathrm{SU}(n) / \mathrm{S}(\mathrm{U}(k) \times \mathrm{U}(n-k))$.

If $(M, g)$ is a compact Kähler manifold with Kähler form $\omega$ and $K$ is a compact connected Lie subgroup of its full isometry group, then the $K$-action is called coisotropic or multiplicity free if the principal $K$-orbits are coisotropic with respect to $\omega$ [7]. Kac [8] and Benson and Ratcliff [1] have given the classification of linear multiplicity free representations, from which one has the full classification of coisotropic actions on $\operatorname{Gr}(k, n)$ for $k=1$, i.e., on the complex projective space. It is therefore natural to investigate this kind of action on complex Grassmannians. Note that, in our analysis, we will always assume $2 \leq k \leq \frac{n}{2}$; if $k>\frac{n}{2}$, we refer to the dual Grassmannian $\operatorname{Gr}\left(k^{\prime}, n\right)$, where $k^{\prime}=n-k \leq \frac{n}{2}$. Our main result is given in the following.

Theorem 1.1. Let $K$ be a compact connected semisimple Lie subgroup of $\mathrm{SU}(n)$, acting nontransitively on the complex Grassmannians

$$
M=G r(k, n)=\mathrm{SU}(n) / \mathrm{S}(\mathrm{U}(k) \times \mathrm{U}(n-k)) .
$$

Then $K$ acts coisotropically on $M$ if and only if its Lie algebra $\mathfrak{k}$ is conjugate to one of the Lie algebras appearing in Table 1.

All the Lie algebras listed in the first column, unless explicitly specified, are meant to be standardly embedded into $\mathfrak{s u}(n)$, e.g., $\mathfrak{s p}(2)+\mathfrak{s u}(n-4) \subset \mathfrak{s u}(4)+$ $\mathfrak{s u}(n-4) \subset \mathfrak{s u}(n)$.

In the following theorem we determine all the minimal nonsemisimple Lie algebras $\mathfrak{k}$ of the compact connected subgroups of $\mathrm{SU}(n)$ acting coisotropically on complex Grassmannians.

Received by the editors April 29, 2003.

2000 Mathematics Subject Classification. Primary 53C55, 57S15.

(C)2004 American Mathematical Society Reverts to public domain 28 years from publication 
TABLE 1.

\begin{tabular}{|c|c|c|}
\hline $\mathfrak{k}$ & $M$ & conditions \\
\hline $\mathfrak{s o}(n)$ & $G r(k, n)$ & \\
\hline $\mathfrak{s p i n}(7)$ & $\operatorname{Gr}(2,8)$ & $\mathfrak{s p i n}(7) \subseteq \mathfrak{s u}(8)$ via spin rep. \\
\hline $\mathfrak{s p}(n)$ & $G r(k, 2 n)$ & \\
\hline $\mathfrak{s p}(n)$ & $G r(k, 2 n+1)$ & $k \neq 3$ \\
\hline $\mathfrak{s p}(2)+\mathfrak{s u}(n-4)$ & $\operatorname{Gr}(4, n)$ & $n \geq 9$ \\
\hline $\mathfrak{s u}(l)+\mathfrak{s u}(p)+\mathfrak{s u}(q)$ & $\operatorname{Gr}(2, n)$ & $p, q>2, n=p+q+l$ \\
\hline $\mathfrak{s u}(l)+\mathfrak{s u}(2)+\mathfrak{s u}(n-l-2)$ & $G r(2, n)$ & $l, n-l>2$ \\
\hline $\mathfrak{s} \mathfrak{u}(l)+\mathfrak{s u}(p)+\mathfrak{s p}(q)$ & $G r(2, n)$ & $q>1, p>2, n=p+2 q+l$ \\
\hline $\mathfrak{s u}(l)+\mathfrak{s u}(n-l-1)$ & $G r(k, n)$ & $n>k+l+1, l>k$ \\
\hline $\mathfrak{s u}(n-1)$ & $G r(k, n)$ & $n \geq 4$ \\
\hline $\mathfrak{s u}(l)+\mathfrak{s u}(n-l)$ & $G r(k, n)$ & $k \neq n-l$ or $k \neq l$ \\
\hline $\mathfrak{s u}(p)+\mathfrak{s u}(q)$ & $G r(k, n)$ & $q>k, p>k, n=p+q+1$ \\
\hline
\end{tabular}

TABLE 2 .

\begin{tabular}{|c|c|c|}
\hline $\mathfrak{k}$ & $M$ & conditions \\
\hline $\mathfrak{z}+\mathfrak{s p}(2)+\mathfrak{s u}(4)$ & $\operatorname{Gr}(4,8)$ & \\
\hline $\mathfrak{z}+\mathfrak{s u}(2)+\mathfrak{s p}(n-1)$ & $G r(2,2 n)$ & $n \geq 3$ \\
\hline $\mathfrak{z}+\mathfrak{s} \mathfrak{u}(3)+\mathfrak{s p}(n-1)$ & $G r(3,2 n+1)$ & $n \geq 3$ \\
\hline $\mathfrak{z}+\mathfrak{s u}(k)+\mathfrak{s u}(k)$ & $G r(k, 2 k)$ & \\
\hline$\overline{\mathfrak{t}_{1}+\mathfrak{s u}(n-2)}$ & $\overline{G r(2, n)}$ & $n>4, \mathfrak{t}_{1}$ maximal torus in $\mathfrak{s u}(2)$ \\
\hline $\mathfrak{a}+\mathfrak{s u}(n-2)$ & $G r(k, n)$ & $k>2, n>4$ \\
\hline $\mathfrak{a}+\mathfrak{s p}(n)$ & $G r(3,2 n+1)$ & \\
\hline $\mathfrak{a}+\mathfrak{s p}(n-1)$ & $G r(2,2 n)$ & $n \geq 2$ \\
\hline $\mathfrak{a}+\mathfrak{s u}(2)$ & $\operatorname{Gr}(2,4)$ & \\
\hline $\mathfrak{a}+\mathfrak{s u}(2)+\mathfrak{s u}(2)+\mathfrak{s u}(2)$ & $G r(2,6)$ & \\
\hline $\mathfrak{a}+\mathfrak{s u}(2)+\mathfrak{s} \mathfrak{u}(2)+\mathfrak{s p}(n-2)$ & $G r(2,2 n)$ & $n>3, n=2 q+4$ \\
\hline $\mathfrak{a}+\mathfrak{s u}(2)+\mathfrak{s p}(p)+\mathfrak{s p}(q)$ & $G r(2,2 n)$ & $p, q \geq 2, n=2 p+2 q+2$ \\
\hline $\mathfrak{a}+\mathfrak{s u}(k)+\mathfrak{s u}(k-1)$ & $G r(k, 2 k)$ & $k \geq 3$ \\
\hline $\mathfrak{a}+\mathfrak{s u}(k)+\mathfrak{s u}(k)$ & $G r(k, 2 k+1)$ & $k \geq 2$ \\
\hline $\mathfrak{a}+\mathfrak{s u}(2)+\mathfrak{s p}(n-1)$ & $G r(2,2 n+1)$ & $n>2$ \\
\hline$\overline{\mathfrak{s u}(n-4)+\mathbb{R}+\mathfrak{s u}(2)+\mathfrak{s u}(2)}$ & $\overline{G r(2, n)}$ & $\overline{c n>6}$ \\
\hline $\mathfrak{s u}(l)+\mathbb{R}+\mathfrak{s u}(2)+\mathfrak{s p}(q)$ & $G r(2, n)$ & $l>2, q \geq 2, n=2 q+2+l$ \\
\hline $\mathfrak{s u}(l)+\mathbb{R}+\mathfrak{s p}(p)+\mathfrak{s p}(q)$ & $G r(2, n)$ & $p, q \geq 2, n=2 p+2 q+l, l>2$ \\
\hline$\overline{\mathfrak{s u}(l)+\mathbb{R}+\mathfrak{s p}(n-1)}$ & $\overline{G r(2,2 n+l-1)}$ & $n>2, l>2$ \\
\hline $\mathfrak{s u}(l)+\mathbb{R}+\mathfrak{s u}(n-l-1)$ & $G r(k, n)$ & $n \geq l+3, l \geq 2, k>n-l$ \\
\hline $\mathbb{R}\left(\frac{n}{k(n-k)}\right)+\mathfrak{s u}(k)+\mathfrak{s u}(n-k-1)$ & $\overline{\overline{G r}(k, n)}$ & $n>2 k+1$ \\
\hline $\mathbb{R}\left(\frac{(k-1) n}{n-1}\right)+\mathfrak{s u}(k-1)+\mathfrak{s u}(n-k)$ & $G r(k, n)$ & $k \geq 3$ \\
\hline $\mathbb{R}\left(\frac{q n}{n-1}\right)+\mathfrak{s u}(p)+\mathfrak{s u}(q)$ & $G r(k, n)$ & $n=p+q+1, p>k, k \geq q+2$. \\
\hline $\mathbb{R}\left(\frac{-n q}{2(n-2)}\right)+\mathfrak{s u}(2)+\mathfrak{s u}(p)+\mathfrak{s p}(q)$ & $\operatorname{Gr}(2, n)$ & $p>2, q>1, n=2+p+2 q$ \\
\hline
\end{tabular}

Theorem 1.2. Let $K$ be a nonsemisimple compact connected Lie subgroup of $\mathrm{SU}(n)$ acting nontransitively on $M$. Then $K$ acts coisotropically on $M$ if and only if its Lie algebra $\mathfrak{k}$, up to conjugation, contains one of the Lie algebras appearing in Table 2, and their semisimple parts coincide.

The notation used in Table 2 is as follows. We denote by $\mathfrak{z}$ the one-dimensional center of the Lie algebra $\mathfrak{h}$ of the group $H=\mathrm{S}(\mathrm{U}(k) \times \mathrm{U}(n-k))$, by a Cartan subalgebra of the centralizer of the semisimple part of $\mathfrak{k}$. In the third block we 
see that the semisimple part of $\mathfrak{k}$ is given by the sum of three ideals $\mathfrak{k}_{1} \subseteq \mathfrak{s u}(l)$, $\mathfrak{k}_{2}+\mathfrak{k}_{3} \subset \mathfrak{s u}(n-l)$ and we denote by $\mathbb{R}$ the centralizer of $\mathfrak{k}_{2}+\mathfrak{k}_{3}$ in $\mathfrak{s u}(n-l)$. In the fourth block $\mathbb{R}$ denotes the centralizer of $\mathfrak{s p}(n-1)(\mathfrak{s u}(n-l-1))$ in $\mathfrak{s u}(2 n-1)$ (resp. $\mathfrak{s u}(n-l))$. Finally, looking at the last block, we consider in the first three cases the product $\mathfrak{z} \times \mathbb{R}$ where $\mathbb{R}$ is the centralizer of the semisimple part in $\mathfrak{s u}(n-1)$. With this notation $\mathbb{R}(\alpha)$ denotes any line in $\mathfrak{z} \times \mathbb{R}$ different from $y=\alpha x$. In the last case we use the same notation, pointing out that here $\mathbb{R}$ is the centralizer of $\mathfrak{s u}(p)+\mathfrak{s p}(q)$ in $\mathfrak{s u}(p+2 q)$.

Victor Kac 8] obtained a complete classification (Tables Ia, Ib, in the Appendix) of irreducible multiplicity free actions $(\rho, V)$. Most of these include a copy of the scalars $\mathbb{C}$ acting on $V$. We will say that a multiplicity free action $(\rho, V)$ of a complex group $G$ is decomposable if we can write $V$ as the direct sum $V=V_{1} \oplus V_{2}$ of proper $\rho(G)$-invariant subspaces in such a way that $\rho(G)=\rho_{1}(G) \times \rho_{2}(G)$, where $\rho_{i}$ denotes the restriction of $\rho$ to $V_{i}$. If $V$ does not admit such a decomposition, then we say that $(\rho, V)$ is an indecomposable multiplicity free action. C. Benson and G. Ratcliff have given the complete classification of indecomposable multiplicity free actions (Tables IIa, IIb in the Appendix). We recall here their theorem (Theorem 2, p. 154 in [1).

Theorem 1.3. Let $(\rho, V)$ be a regular representation of a connected semisimple complex algebraic group $G$ and decompose $V$ as a direct sum of $\rho(G)$-irreducible subspaces, $V=V_{1} \oplus V_{2} \oplus \cdots \oplus V_{r}$. The action of $\left(\mathbb{C}^{*}\right)^{r} \times G$ on $V$ is an indecomposable multiplicity free action if and only if either

(1) $r=1$ and $\rho(G) \subseteq \mathrm{GL}(V)$ appears in Table Ia (see the Appendix);

(2) $r=2$ and $\rho(G) \subseteq \mathrm{GL}\left(V_{1}\right) \times \mathrm{GL}\left(V_{2}\right)$ appears in Tables IIa and IIb (see the Appendix).

In [1] conditions are also given under which one can remove or reduce the copies of the scalars preserving the multiplicity free action. Obviously, if an action is coisotropic, it continues to be coisotropic also when this action includes another copy of the scalars. We will call minimal those coisotropic actions in which the scalars, if they appear, cannot be reduced.

Let $K$ be a compact group acting isometrically on a compact Kähler manifold $M$. We say that $M$ is $K^{\mathbb{C}}$-almost homogeneous if $K^{\mathbb{C}}$ has an open orbit in $M$. If all Borel subgroups of $K^{\mathbb{C}}$ act with an open orbit on $M$, then the $K^{\mathbb{C}}$-open orbit $\Omega$ is called a spherical homogeneous space and $M$ is called a spherical embedding of $\Omega$. We will briefly recall some results that will be used in the sequel.

Theorem 1.4 ([7]). Let $M$ be a connected compact Kähler manifold with an isometric action of a connected compact group $K$ that is also Poisson. Then the following conditions are equivalent:

(i) The $K$-action is coisotropic.

(ii) The cohomogeneity of the $K$ action is equal to the difference between the rank of $K$ and the rank of a regular isotropy subgroup of $K$.

(iii) The moment map $\mu: M \rightarrow \mathfrak{k}^{*}$ separates orbits.

(iv) The Kähler manifold $M$ is projective algebraic, $K^{\mathbb{C}}$-almost homogeneous and a spherical embedding of the open $K^{\mathbb{C}}$-orbit.

As an immediate consequence of the above theorem one can deduce, under the same hypotheses on $K$ and $M$, the following two simple facts that will be frequently used in our classification. 
1. Let $p$ be a fixed point on $M$ for the $K$-action, or $K p$ a complex $K$-orbit. Then the $K$-action is coisotropic if and only if the slice representation is coisotropic (see [7], p. 274).

2. Dimensional condition. If $K$ acts coisotropically on $M$, the dimension of a Borel subgroup $B$ of $K^{\mathbb{C}}$ is not less than the dimension of $M$.

A relatively large class of coisotropic actions is provided by polar ones. We recall here that an isometric action of a group $K$ is called polar on $M$ if there exists a properly embedded submanifold $\Sigma$ which meets every $K$-orbit and is orthogonal to the $K$-orbits in all common points. Such a submanifold is called a section, and, if it is flat, the action is called hyperpolar.

A result due to Hermann states that given a compact Lie group $K$ and two symmetric subgroups $H_{1}, H_{2} \subseteq K$, then $H_{i}$ acts hyperpolarly on $K / H_{j}$ for $i, j \in 1,2$. Therefore, given an Hermitian symmetric space as

$$
G r(k, n)=\mathrm{SU}(n) / \mathrm{S}(\mathrm{U}(k) \times \mathrm{U}(n-k))
$$

it is clear that subgroups like $\mathrm{SO}(n), \mathrm{Sp}(n)$ and $\mathrm{S}(\mathrm{U}(l) \times \mathrm{U}(n-l))$ will act on $\operatorname{Gr}(k, n)$ hyperpolarly; hence, since for 12 a polar action on an irreducible compact homogeneous Kähler manifold is coisotropic, these actions are coisotropic.

Once we have determined the complete list of coisotropic actions on $\operatorname{Gr}(k, n)$, in Table 2, we have also investigated which ones are polar. Dadok [3, Heintze and Eschenburg 5] have classified the irreducible polar linear representations, while I. Bergmann [2] has found all the reducible polar ones. Using their results we determine in Section 3 the polar actions on complex Grassmannians. We get the following.

Theorem 1.5. Let $K$ be a compact connected Lie subgroup of $\mathrm{SU}(n)$ acting nontransitively on the complex Grassmannians

$$
M=G r(k, n)=\mathrm{SU}(n) / \mathrm{S}(\mathrm{U}(k) \times \mathrm{U}(n-k)) .
$$

Then $K$ acts polarly on $M$ if and only if its Lie algebra $\mathfrak{k}$ is conjugate to one of the Lie algebras appearing in Table 3 , where $\mathbb{R}$ is the centralizer of $\mathfrak{s u}(n-l)+\mathfrak{s u}(l)$ in $\mathfrak{s u}(n)$. In particular, the $K$-action is hyperpolar.

TABLE 3.

\begin{tabular}{|c|c|c|}
\hline $\mathfrak{k}$ & $M$ & conditions \\
\hline \hline $\mathfrak{s o}(n)$ & $G r(k, n)$ & \\
\hline $\mathfrak{s p}(n)$ & $G r(k, 2 n)$ & \\
\hline $\mathbb{R}+\mathfrak{s u}(l)+\mathfrak{s u}(n-l)$ & $G r(k, n)$ & \\
\hline $\mathfrak{s u}(l)+\mathfrak{s u}(n-l)$ & $G r(k, n)$ & $l \neq n-l$ \\
\hline
\end{tabular}

An interesting consequence of this classification is that polar actions on complex Grassmannians are hyperpolar. This is in contrast with complex projective spaces or, more generally, to rank one symmetric spaces that admit many polar actions that are not hyperpolar [13].

Here we briefly explain our method in order to prove our main theorem. Thanks to Theorem 1.4 (iv) we have that if $K$ is a subgroup of a compact Lie group $L$ such that $K$ acts coisotropically on $M$, then so does $L$. As a consequence, in order to classify coisotropic actions on $\operatorname{Gr}(k, n)$, one may suggest a sort of "telescopic" procedure by restricting to maximal subgroups $K$ of $\mathrm{SU}(n)$, hence passing to maximal 
subgroups that give rise to coisotropic actions and so on. The same technique can be used in order to obtain a complete classification of polar and coisotropic actions on irreducible Hermitian symmetric spaces of compact type; this will be given in a forthcoming paper.

In the Appendix we give the complete list of maximal subgroups $K$ of $\mathrm{SU}(n)$.

This paper is organized as follows. In Section 2 we prove Theorem 1.1 and Theorem 1.2. We have divided this section into five subsections in each of which we analyze separately one of the maximal subgroups of $\mathrm{SU}(n)$. In the third section we give the proof of Theorem 1.5 .

We enclose, in the Appendix, the tables of irreducible and reducible linear multiplicity free representations (Tables Ia, Ib and Tables IIa, IIb respectively), the table of maximal subgroups of $\mathrm{SU}(n)$ and $\operatorname{Sp}(n)$ (Tables III, IV) and the table of reduced prehomogeneous triplets (Table V), since we will frequently refer to them in our analysis.

\section{Proof of Theorem 1.1 and Theorem 1.2}

In the following subsections we will go through all maximal subgroups $K$ of $\mathrm{SU}(n)$ according to Table III in the Appendix.

2.1. The case $K=\mathrm{SO}(n)$. Note that $\mathrm{SO}(n)$ is a symmetric subgroup of $\mathrm{SU}(n)$. The action is polar, hence coisotropic on complex Grassmannians. We can now investigate the behaviour of subgroups of $\mathrm{SO}(n)$. Take a $k$-plane $\pi$ in $\mathbb{R}^{n}$. The $\mathrm{SO}(n)$-orbit of $\pi$ is a real nonoriented Grassmannian. Note that the orbit coincides with the set of fixed points of the conjugation, which is an antiholomorphic isometry on $\operatorname{Gr}(k, n)$, hence the orbit is totally real in $\operatorname{Gr}(k, n)$. The following proposition is easy to prove using well-known properties of the moment map.

Proposition 2.1. Let $K$ be a semisimple Lie group which acts on a Kähler manifold $M$ with a totally real orbit $K p$ and moment map $\mu$. Then $\mu(K p)=0$.

In the sequel we will identify $\mathfrak{k} \cong \mathfrak{k}^{*}$ by means of an $\operatorname{Ad}(K)$-invariant inner product on $\mathfrak{k}$.

From the previous proposition we deduce that 0 belongs to the image of the moment map $\mu: G r(k, n) \rightarrow \mathfrak{s o}(\mathfrak{n})$. Now take the subgroup $K^{\prime}$ of $\operatorname{SO}(n)$. Obviously its image via the moment map $\mu^{\prime}$ of $K^{\prime}$, given by the composition of $\mu$ with the projection map $\mathfrak{k} \rightarrow \mathfrak{k}^{\prime}$, contains 0 . If $K^{\prime}$ acts coisotropically, then $\mu^{\prime}$ separates, by Theorem 1.4 (iii), the orbits and therefore $\mu^{\prime-1}(0)$ coincides with the real nonoriented Grassmannian. The problem is then restricted to the analysis of those subgroups $K^{\prime}$ of $\mathrm{SO}(n)$ that act transitively on the real Grassmannian and coisotropically on the complex $G r(k, n)$. The first problem was solved by Onishchick in 11 .

We have to distinguish two cases, namely $k=2$ and $k>2$.

In the first case we have to consider the subgroups $G_{2}$ and $\operatorname{Spin}(7)$ of $\mathrm{SO}(7)$ and $\mathrm{SO}(8)$ respectively. The first one, $G_{2}$, cannot act coisotropically on $\operatorname{Gr}(2,7)$ because the Borel subgroup of $G_{2}^{\mathbb{C}}$ has complex dimension $8<\operatorname{dim} \operatorname{Gr}(2,7)$. Spin $(7)$ acts, instead, coisotropically on $\operatorname{Gr}(2,8)$. Note that the totally real orbit is, in this case, the nonoriented real Grassmannian $G r_{\mathbb{R}}(2,8)$ and $\mathrm{U}(3)$ is the isotropy subgroup of $\operatorname{Spin}(7)$. It is well known that the cohomogeneity $\operatorname{chm}(\operatorname{Gr}(2,8), \operatorname{Spin}(7))=$ $\operatorname{chm}($ Slice, $\mathrm{U}(3))$; now, the slice representation of $\mathrm{U}(3)$ on $\operatorname{Gr}(2,8)$ can be deduced 
immediately from the isotropy representation of $\mathrm{U}(3)$ that is given by $\mathbb{C}^{3} \oplus\left(\mathbb{C}^{3}\right)^{*}=$ $\mathbb{C}^{3} \oplus \Lambda^{2}\left(\mathbb{C}^{3}\right)$, since the orbit is totally real. Furthermore, observe that $\operatorname{rk}(\operatorname{Spin}(7))=$ $\operatorname{rk}(\mathrm{U}(3))$ and the same holds for the principal isotropy subgroups; hence, by Theorem 1.4 (ii), the $\operatorname{Spin}(7)$-action is coisotropic if and only if the U(3)-action is coisotropic. Now, looking at the list of Benson and Ratcliff (Table IIa, Appendix), we conclude that the action of $\mathrm{U}(3)$ is coisotropic since the scalars act on $\mathbb{C}^{3} \oplus\left(\mathbb{C}^{3}\right)^{*}$ as $z\left(v_{1}, v_{2} \wedge v_{3}\right)=\left[z v_{1}, z^{2}\left(v_{2} \wedge v_{3}\right)\right]$ and $a=1 \neq-b=-2$.

In the second case, when $k>2$, only $K^{\prime}=\operatorname{Spin}(7)$ acts transitively on the real nonoriented Grassmannian of 3-planes in $\mathbb{R}^{8}$. As for $G_{2}$, the Borel subgroup of $\operatorname{Spin}(7)$ is too small: it has, in fact, complex dimension 12 which is smaller than the complex dimension of $\operatorname{Gr}(3,8)$.

This completes the analysis; we have thus proved that the only subgroups of $\mathrm{SO}(n)$ which act coisotropically on some Grassmannian $G r(k, n)$ are $\mathrm{SO}(n)$ and $\operatorname{Spin}(7) \subset \mathrm{SO}(8)$.

2.2. The case $K=\mathrm{SU}(p) \otimes \mathrm{SU}(q)$. For simplicity we will denote the group $\mathrm{SU}(p) \times$ $\mathrm{SU}(q)$ by $K \subseteq \mathrm{SU}(n)$, with $n=p q$.

In this case we will determine the slice representation on a complex orbit, showing that it is not coisotropic. We recall the following simple lemma.

Lemma 2.1. Let $\rho: G \rightarrow \mathrm{GL}(V)$ be a linear coisotropic representation. If $V$ splits as the direct sum $V_{1} \oplus V_{2} \oplus \ldots \oplus V_{l}$ of irreducible submodules, then $\rho(G)$ restricted on each $V_{i}$ is coisotropic.

We have to distinguish two different cases:

(1) Either $p$ or $q$ is bigger than $k$. We can assume, for example, that $p \geq$ $k$. We choose a $k$-dimensional subspace $W \subseteq \mathbb{C}^{p}$ and a one-dimensional $V \subseteq \mathbb{C}^{q}$. The stabilizer $K_{\pi}$, where $\pi$ is the $k$-plane $W \otimes V \subseteq \mathbb{C}^{p} \otimes \mathbb{C}^{q}$, is $\mathrm{S}(\mathrm{U}(k) \times \mathrm{U}(p-k)) \times \mathrm{S}(\mathrm{U}(1) \times \mathrm{U}(q-1))$; it is easy to see that the tangent space $T_{\pi} G_{r}(k, p)=\pi^{*} \otimes \pi^{\perp}$ splits, as a $K_{\pi^{-}}$-module, as

$\left(W^{*} \otimes W^{\perp}\right) \oplus\left(V^{*} \otimes V^{\perp}\right) \oplus\left(\mathfrak{s l}(W) \otimes V^{*} \otimes V^{\perp}\right) \oplus\left(W^{*} \otimes W^{\perp} \otimes V^{*} \otimes V\right)$

where $W^{\perp}, V^{\perp}$ are the orthogonal complements of $W, V$ in $\mathbb{C}^{p}, \mathbb{C}^{q}$ respectively. Here we have used the fact that $W^{*} \otimes W=\mathbb{C} \oplus \mathfrak{s l}(W)$ as $U(k)$ modules. The first two factors can be identified with the tangent space at $\pi$ of the $K$-orbit $K \pi$, which is therefore complex; on the other hand, the summand $\mathfrak{s l}(W) \otimes V^{*} \otimes V^{\perp}$ does not appear in Kac's list, so that the $K$-action is not coisotropic, thanks to Lemma 2.1.

(2) $p$ and $q$ are smaller than $k$. Clearly $k=m p+l$ where $m<q$ and $l<p$. Choose an $l$-dimensional subspace $W \subseteq \mathbb{C}^{p}$, an $m$-dimensional $V \subseteq \mathbb{C}^{q}$, and $v_{m+1} \in \mathbb{C}^{q} \backslash V$. We consider a $k$-complex plane $\pi=\left(\mathbb{C}^{p} \otimes V\right) \oplus\left(W \otimes v_{m+1}\right)$; it is easy to check that the $K$-orbit

$$
K \pi \cong G r(l, p) \times \mathrm{SU}(q) / \mathrm{S}(\mathrm{U}(1) \times \mathrm{U}(m) \times \mathrm{U}(q-m-1))
$$

is complex. Following the same procedure used in the previous case, with slightly heavier computations, we determine the slice summand $\mathfrak{s t}\left(W^{\perp}\right) \otimes$ $V^{*} \otimes v_{m+1}$, on which $A d(\mathrm{U}(l)) \otimes \mathrm{S}(\mathrm{U}(m) \times \mathrm{U}(1))$ acts; this action does not appear in Kac's list. Hence, using again Lemma 2.1, we can conclude that the $\mathrm{SU}(p) \otimes \mathrm{SU}(q)$-action cannot be coisotropic. 
2.3. The case of a simple Lie group $K=H$ such that $\rho(H)$ is an irreducible representation of complex type. This case can be analyzed using the work of Sato and Kimura [14]. Throughout this section we will identify the fundamental highest weights $\Lambda_{l}$ with the corresponding irreducible representations.

We recall here that, given $G$ a connected complex linear algebraic group, and $\rho$ a rational representation of $G$ on a finite dimensional complex vector space $V$, such a triplet $(G, \rho, V)$ is prehomogeneous if $V$ has a Zariski dense $G$-orbit.

Definition 2.1. Two triplets $(G, \rho, V)$ and $\left(G^{\prime}, \rho^{\prime}, V^{\prime}\right)$ are called equivalent if there exist a rational isomorphism $\sigma: \rho(G) \rightarrow \rho^{\prime}\left(G^{\prime}\right)$ and an isomorphism $\tau: V \rightarrow V^{\prime}$, both defined over $\mathbb{C}$ such that the diagram is commutative for all $g \in G$.

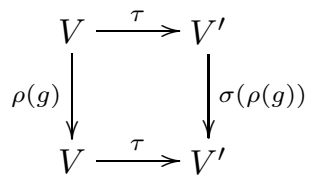

This equivalence relation will be denoted by $(G, \rho, V) \cong\left(G^{\prime}, \rho^{\prime}, V^{\prime}\right)$.

We say that two triplets $(G, \rho, V)$ and $\left(G^{\prime}, \rho^{\prime}, V^{\prime}\right)$ are castling transforms of each other when there exist a triplet $(\tilde{G}, \tilde{\rho}, V(m))$ and a positive number $n$ with $m>n \geq 1$ such that

$$
(G, \rho, V) \cong\left(\tilde{G} \times \mathrm{SL}(n), \tilde{\rho} \otimes \Lambda_{1}, V(m) \otimes V(n)\right)
$$

and

$$
\left(G^{\prime}, \rho^{\prime}, V^{\prime}\right) \cong\left(\tilde{G} \times \mathrm{SL}(m-n), \tilde{\rho}^{*} \otimes \Lambda_{1}, V(m)^{*} \otimes V(m-n)\right),
$$

where $\tilde{\rho}^{*}$ is the contragradient representation of $\tilde{\rho}$ on the dual vector space $V(m)^{*}$ of $V(m)$. We recall that $V(n)$ is a complex vector space of dimension $n$. A triplet $(G, \rho, V)$ is called reduced if there is no castling transform $\left(G^{\prime}, \rho^{\prime}, V^{\prime}\right)$ with $\operatorname{dim} V^{\prime}<$ $\operatorname{dim} V$. We give here a useful lemma that permits us to find relations between coisotropic actions on Grassmannians, which must have an open dense orbit, and prehomogeneous triplets. This can be easily proved as a consequence of Lemma 5 and Proposition 7, p. 37 in section 3 of [14].

Lemma 2.2. Let $G$ be any complex, connected Lie group. $G$ acts with an open dense orbit on $G r(k, n)$ if and only if $G \times G L(k)$ acts with an open dense orbit on $\mathbb{C}^{n} \otimes \mathbb{C}^{k}$, i.e., $\left(G \times \mathrm{GL}(k), \rho, \mathbb{C}^{n} \otimes \mathbb{C}^{k}\right)$ is a prehomogeneous triplet.

Let $H$ be a simple group; denote with $d$ the degree of $\rho$ and with $V(d)$ its representation space. We are looking for simple groups $H$, whose representation is irreducible and of complex type, that act coisotropically on complex Grassmannians $\operatorname{Gr}(k, d)$. Recall that one can restrict the analysis to $2<k \leq \frac{d}{2}$; in fact, for $k>\frac{d}{2}$ one can take the dual Grassmannian $G r(d-k, d)$. With these notations we state and prove the following.

Lemma 2.3. If $\mathfrak{h} \neq A_{n}$, then the triplet $\left(H \times \mathrm{GL}(k), \rho \otimes \Lambda_{1}, V(d) \otimes V(k)\right)$ is reduced.

Proof. Suppose, by contradiction, that the triplet is not reduced. Then there would exist two triplets $(\tilde{G}, \tilde{\rho}, V(m))$ and $\left(G^{\prime}, \rho^{\prime}, V^{\prime}\right)$, with $\operatorname{dim} V^{\prime}<\operatorname{dim}(V(d) \otimes V(k))$, such that

(a) $\left(H \times \mathrm{GL}(k), \rho \otimes \Lambda_{1}, V(d) \otimes V(k)\right) \cong\left(\tilde{G} \times \mathrm{SL}(n), \tilde{\rho} \otimes \Lambda_{1}, V(m) \otimes V(n)\right)$; 
(b) $\left(G^{\prime}, \rho^{\prime}, V^{\prime}\right) \cong\left(\tilde{G} \times \mathrm{SL}(m-n), \tilde{\rho}^{*} \otimes \Lambda_{1}, V(m)^{*} \otimes V(m-n)\right)$.

Switching to the Lie algebra level we get, from the first condition $\mathfrak{h}+\mathfrak{g l}(k) \cong$ $d \tilde{\rho}(\tilde{\mathfrak{g}})+\mathfrak{s l}(n)$ which implies, since $\mathfrak{h} \neq A_{n}$, that $n=k$ and $m=d$, and, from the second, we get $\operatorname{dim} V^{\prime}=d(d-k)$. This gives rise to a contradiction since $\operatorname{dim} V^{\prime} \geq \operatorname{dim}(V(d) \otimes V(k))$.

Sato and Kimura have completely classified the prehomogeneous reduced triplets. Hence the problem, if $\mathfrak{h} \neq A_{n}$, boils down to see whether the triplet $(H \times \operatorname{GL}(k)$, $\left.\rho \otimes \Lambda_{1}, V(d) \otimes V(k)\right)$ appears in the list of Sato Kimura (see Table V, Appendix). If it is not the case, the $H$-action is not coisotropic.

We find, for $k=2$, the triplet $\left(\operatorname{Spin}(7, \mathbb{C}) \times \mathrm{GL}(2)\right.$, spin. representation $\otimes \Lambda_{1}$, $\left.\mathbb{C}^{8} \otimes \mathbb{C}^{2}\right)$; hence $\operatorname{Spin}(7)$ has an open orbit on $\operatorname{Gr}(2,8)$; it has already been studied as a subgroup of $\mathrm{SO}(8)$. This action is coisotropic.

The other groups, arising from the triplets $(\operatorname{Spin}(10) \times \mathrm{GL}(2)$, half - spin. representation $\left.\otimes \Lambda_{1}, \mathbb{C}^{16} \otimes \mathbb{C}^{2}\right),\left(G_{2} \times \mathrm{GL}(2), \Lambda_{2} \otimes \Lambda_{1}, \mathbb{C}^{7} \otimes \mathbb{C}^{2}\right)$ and $\left(E_{8}^{\mathbb{C}} \times \mathrm{GL}(2)\right.$, $\left.\Lambda_{1} \otimes \Lambda_{1}, \mathbb{C}^{27} \otimes \mathbb{C}^{2}\right)$ can be excluded thanks to the dimensional condition.

For $k>2$ we find again $\operatorname{Spin}(7)$ on $\operatorname{Gr}(3,8)$, and $\operatorname{Spin}(10)$ on $\operatorname{Gr}(3,16)$ and they are excluded for the same dimensional reason.

Note that in the list of Sato and Kimura the group $\operatorname{Sp}(n)$ appears; this case will be studied separately in the next subsection.

Now, suppose $\mathfrak{h}=A_{n}$. We impose the dimensional condition. Denote with $\mathfrak{b}$ the Lie algebra of the Borel subgroup of $H^{\mathbb{C}}$. We get the following inequality:

$$
\operatorname{dim}_{\mathbb{C}} \mathfrak{b} \geq \operatorname{dim}_{\mathbb{C}} G r(k, d)
$$

that, when $\mathfrak{h}=A_{n}$, becomes

$$
\frac{(n-1)(n+2)}{2 k}+k \geq d
$$

Using Young diagrams we can write down the degree of each representation.

To solve this case we distinguish the case $k=2$ and $k \neq 2$. For $n=2$ and $k=2$ the fact that only $\Lambda_{1}$ and $2 \Lambda_{1}$ are admissible is straightforward. If $k=2$, and $n \geq 3$, then it is easy to prove that $d<\frac{1}{2} n(n+1)<n^{2}$, hence, using Proposition 7 and Corollary 8 (p. 45, section 3 of [14]), we have only to investigate the representations $\Lambda_{1}, \Lambda_{2}, \Lambda_{n-1}, \Lambda_{n-2}, n=3,4$. Note that $\Lambda_{1}, \Lambda_{n-1}$ correspond to the transitive action of $\mathrm{SU}(n)$ on $\operatorname{Gr}(k, n)$. We then find the following two triplets: when $n=$ 2, $\left(\mathrm{SL}(2), 2 \Lambda_{1}, S^{2}\left(\mathbb{C}^{2}\right)\right)$ which corresponds to a coisotropic action on the complex projective space and for $n=4$, we get $\left(\mathrm{SL}(4), \Lambda_{2}, \mathbb{C}^{6}\right)$ that must be excluded since it is of real type.

In the general case, when $2<k \leq \frac{d}{2}$, we can do analogous calculations excluding all the simple groups which act nontransitively, i.e., which have representations different from $\Lambda_{1}$ and $\Lambda_{n-1}$. Indeed, we note that

$$
d \leq \frac{(n-1)(n+2)}{2 k}+k \leq \frac{(n-1)(n+2)}{6}+\frac{d}{2},
$$

hence

$$
d \leq \frac{(n-1)(n+2)}{3} .
$$

Consider the case $\rho=\Lambda_{2}$ separately. The inequality (2) holds for $n \leq 4$ and the corresponding cases are $\left(\mathrm{SL}(3), \Lambda_{2}, \mathbb{C}^{3}\right)$, which corresponds to the transitive action 
of $\mathrm{SL}(3)$ on $\operatorname{Gr}(1,3)$, and to $\left(\mathrm{SL}(4), \Lambda_{2}, \mathbb{C}^{6}\right)$ that can be excluded since it is of real type.

We then study the general case, analyzing $d\left(\Lambda_{l}\right)$, where $2<l \leq \frac{n}{2}$ (if $l>\frac{n}{2}$ we can consider the dual representation which shares the same behavior). We shall prove that the inequality (2) does not hold if $\rho \neq \Lambda_{1}, \Lambda_{n-1}$. Indeed, it becomes for $\rho=\Lambda_{l}$,

$$
3 n(n-2) \cdots(n-l+1) \leq(n+2) l !
$$

with the condition $n \geq 2 l>4$. This can never be satisfied.

Now, observing that the degree $d\left(\sum_{i} m_{i} \Lambda_{i}\right)$ increases as $m_{i}$ increases it is sufficient to prove that $m \Lambda_{1}, \Lambda_{1}+\Lambda_{2}$ and $\Lambda_{1}+\Lambda_{n-1}$ do not satisfy condition (2) in order to get our assertion. This is a straightforward calculation using Young diagrams.

We conclude that no simple group $H$ with $\rho(H) \neq \Lambda_{1}, \Lambda_{n-1}$ gives rise to a coisotropic action, except for $\operatorname{Spin}(7) \subset \mathrm{SO}(8)$ and $\mathrm{Sp}(n) \subset \mathrm{SU}(2 n)$.

2.4. The case $K=\operatorname{Sp}(n)$. We now consider the case $K=\operatorname{Sp}(n) \subseteq \mathrm{SU}(2 n)$ acting on the Grassmannian $\operatorname{Gr}(k, 2 n)$. Note that $\operatorname{Sp}(n)$ is a symmetric subgroup of $\mathrm{SU}(2 n)$; the action is then polar, hence coisotropic on complex Grassmannians. We will now go through the maximal subgroups $K^{\prime}$ of $\operatorname{Sp}(n)$ (see Table IV in the Appendix, for the complete list).

(i) $K^{\prime}=\mathrm{U}(n)$. We recall that the subgroup $K^{\prime}=\mathrm{U}(n)$ acts on $\mathbb{C}^{2 n}$ reducibly, namely as $\rho_{n} \oplus \rho_{n}^{*}$ where $\rho_{n}$ denotes the standard representation of $U(n)$ in $\mathbb{C}^{n}$. We consider a $k$-dimensional subspace $\pi \subseteq \mathbb{C}^{n}$, since we can always suppose $k \leq n$; then $K^{\prime} \pi=\mathrm{U}(n) / \mathrm{U}(k) \times \mathrm{U}(n-k)$ and $T_{\pi} G r(k, 2 n)$ splits, as a $\mathrm{U}(k) \times \mathrm{U}(n-k)$ module, as $S^{2}\left(\pi^{*}\right) \oplus \Lambda^{2}\left(\pi^{*}\right) \oplus \pi^{*} \otimes \mathbb{C}^{n-k} \oplus \pi^{*} \otimes \mathbb{C}^{n-k^{*}}$. This means that $K^{\prime} \pi$ is a complex orbit with slice representation $S^{2}\left(\pi^{*}\right) \oplus \Lambda^{2}\left(\pi^{*}\right) \oplus \pi^{*} \otimes \mathbb{C}^{n-k^{*}}$ which is not coisotropic by [1].

(ii) $K^{\prime}=\mathrm{SO}(p) \otimes \mathrm{Sp}(q), p q=n, p \geq 3, q \geq 1$. Clearly,

$$
S O(p) \otimes \mathrm{Sp}(q) \subset \mathrm{SU}(p) \otimes \mathrm{SU}(2 q),
$$

hence the action cannot be coisotropic as the action of $\mathrm{SU}(p) \otimes \mathrm{SU}(2 q)$ is not (section 2.2).

(iii) $K^{\prime}=\rho(H), H$ simple, $\rho \in \operatorname{Irr}_{\mathbb{H}}, \operatorname{deg} \rho=n$. With the same estimates on the degree of the representation that we have used in the previous paragraph, it is easy to prove that these groups cannot acts coisotropically on the complex Grassmannians.

(iv) $K^{\prime}=\operatorname{Sp}(l) \times \operatorname{Sp}(n-l)$. First of all we shall give a complex orbit for the $\operatorname{Sp}(n)$ action. We recall that $k \leq n$. We identify the $\operatorname{Sp}(n)$-module $\mathbb{H}^{n}$ with $\mathbb{C}^{2 n}=\mathbb{C}^{n} \oplus \mathbb{C}^{n}$ endowed with the anti-holomorphic map $J \in \mathbb{C}^{2 n}$ commuting with the $\operatorname{Sp}(n)$-action. If we select a $k$-plane $\pi \subseteq \mathbb{C}^{n}$, it is not difficult to see that the stabilizer $\operatorname{Sp}(n)_{\pi}$ is given by $\mathrm{U}(k) \times \operatorname{Sp}(n-k)$. We have that $\pi^{\perp}=J \pi \oplus W$, where $W$ is a quaternionic subspace of complex dimension $2(n-k)$; note that $J \pi=\pi^{*}$ as $\mathrm{U}(k)$-modules. The tangent space $T_{\pi} G r(k, 2 n)$ splits into irreducible complex $\operatorname{Sp}(n)_{\pi^{-} \text {-submodules as }}$

$$
\pi^{*} \otimes \pi^{\perp}=S^{2} \pi^{*} \oplus \Lambda^{2} \pi^{*} \oplus\left(\pi^{*} \otimes W\right),
$$

while, on the other hand, the isotropy representation of $\operatorname{Sp}(n) / \operatorname{Sp}(n)_{\pi}$ is given by $S^{2} \pi^{*} \oplus L$, where $L=\mathbb{H}^{k} \otimes_{\mathbb{H}} \mathbb{H}^{n-k}$ as real submodules. From this we see that the slice is isomorphic to $\Lambda^{2} \pi^{*}$ and that the orbit $\operatorname{Sp}(n) \pi$ is complex. Note that this action appears in [8], this is another way to see that $\operatorname{Sp}(n)$ acts on complex Grassmannians coisotropically. 
We now consider $K^{\prime}=\operatorname{Sp}(l) \times \operatorname{Sp}(n-l)$. We take $k_{1}, k_{2}$ such that $k_{1} \leq l, k_{2} \leq$ $n-l$ and $k=k_{1}+k_{2}$. Let $\pi_{1}$ and $\pi_{2}$ be two complex $k_{1^{-}}$and $k_{2}$-planes in $\mathbb{C}^{2 l}$ and $\mathbb{C}^{2 n-2 l}$ respectively, such that the orbits in $G r\left(k_{1}, 2 l\right)$ and $G r\left(k_{2}, 2 n-2 l\right)$ resp. are complex. Let $\pi=\pi_{1} \oplus \pi_{2}$ be the $k$-complex plane in $\mathbb{C}^{2 n}$. It is easy to see that the stabilizer $K_{\pi}^{\prime}$ of the $k$-plane $\pi$ is $\mathrm{U}\left(k_{1}\right) \times \mathrm{Sp}\left(l-k_{1}\right) \times \mathrm{U}\left(k_{2}\right) \times \operatorname{Sp}\left(n-l-k_{2}\right)$. With the same notation that we have used before, it is easy to check that the slice representation is given by

$$
\Lambda^{2}\left(\pi_{1}\right) \oplus \Lambda^{2}\left(\pi_{2}\right) \oplus\left(\pi_{1}^{*} \otimes \pi_{2}^{*}\right) \oplus\left(\pi_{1}^{*} \otimes \pi_{2}^{*}\right) \oplus\left(\pi_{1}^{*} \otimes W_{2}\right) \oplus\left(\pi_{2}^{*} \otimes W_{1}\right)
$$

and looking at Tables IIa, IIb we can conclude that this action is not coisotropic on the complex Grassmannians.

Note that if we consider the $\operatorname{Sp}(n)$-action on the complex Grassmannians, with $k$ even, the orbit of the $k$-plane $\pi$ generated by $v_{1}, v_{2} \cdots v_{k}$ and $J v_{1}, J v_{2} \cdots J v_{k}$, is totally real (for $k=2$ it is $\mathbb{H} \mathbb{P}^{n-1}=\operatorname{Sp}(n) / \operatorname{Sp}(1) \times \operatorname{Sp}(n-1)$ ). We may restrict our analysis, as in the $\mathrm{SO}(n)$ case, to those subgroups of $\mathrm{Sp}(n)$ that act transitively on the totally real orbit, and coisotropically on the complex Grassmannians. In [11] one can find that no subgroups of $\operatorname{Sp}(n)$ can act transitively on the totally real orbit. This gives another proof, when $k$ is even, of the fact that no subgroup of $\operatorname{Sp}(n)$ can act coisotropically on $\operatorname{Gr}(k, n)$.

2.5. The reducible case. We can investigate directly the subgroups $L$ of $K=$ $\mathrm{S}(\mathrm{U}(l) \times \mathrm{U}(n-l))$ because $K$ acts coisotropically on the complex Grassmannians. Recall that, in the sequel, we always assume $1<k \leq \frac{n}{2}$. If $k>\frac{n}{2}$, we refer to the dual Grassmannian $G r\left(k^{\prime}, n\right)$, where $k^{\prime}=n-k \leq \frac{n}{2}$.

With this assumption we divide the reducible case in three paragraphs: in the first one we will analyze the case $k=l$, namely when the group $K$ has a fixed point in $\operatorname{Gr}(k, n)$; in the second we suppose $k<l, n-l$, finally in the third one we complete the analysis studying the case $k<l, k>n-l$. We will also, for simplicity switch to the Lie algebra level, denoting by $\mathfrak{z}$ the one-dimensional center of the Lie algebra $\mathfrak{k}$ of $K$.

2.5.1. The fixed point case. Consider $L \subseteq K=\mathrm{S}(\mathrm{U}(k) \times \mathrm{U}(n-k))$ and $\pi=\mathbb{C}^{k}$ a fixed $k$-plane. Let $\mathfrak{l}$ be the Lie algebra of $L, \mathfrak{l} \subseteq \mathfrak{s u}(k)+\mathfrak{s u}(n-k)+\mathbb{R}=\mathfrak{k}$. Suppose $\mathfrak{l}$ acts coisotropically. We consider the projections $\rho_{1}: \mathfrak{l} \longrightarrow \mathfrak{s u}(k), \rho_{2}: \mathfrak{l} \longrightarrow \mathfrak{s u}(n-k)$ and we put $\mathfrak{l}_{i}=\rho_{i}(\mathfrak{l})$. This means that $\mathfrak{z}+\mathfrak{l}_{1}+\mathfrak{l}_{2}$ acts coisotropically. We distinguish some cases according to the irreducibility of $\mathfrak{l}_{i}$ on the slice $S$ at $\pi=\mathbb{C}^{k}$, that is given by $\left(\mathbb{C}^{k}\right)^{*} \otimes \mathbb{C}^{n-k}$. In the sequel we refer to Tables Ib, IIa and IIb in the Appendix, for all the conditions under which one can remove the scalars preserving the multiplicity free action.

(1) $\mathfrak{l}_{1}$ and $\mathfrak{l}_{2}$ act both irreducibly on $\left(\mathbb{C}^{k}\right)^{*}$ and $\mathbb{C}^{n-k}$ respectively. Since $\mathfrak{l}_{i} \subseteq$ $\mathfrak{s u}(k)$ or $\mathfrak{s u}(n-k)$, we have that $\mathfrak{l}_{i}$ are centerless. Hence $\mathfrak{l}_{1}+\mathfrak{l}_{2}$ is semisimple and acts irreducibly on $\left(\mathbb{C}^{k}\right)^{*} \otimes \mathbb{C}^{n-k}$. From Table Ia we get:

\begin{tabular}{|c|c|c|c|}
$n$. & $\mathfrak{l}_{1}$ & $\mathfrak{l}_{2}$ & conditions \\
\hline 1. & $\mathfrak{s u}(k)$ & $\mathfrak{s u}(n-k)$ & \\
2. & $\mathfrak{s p}(2)$ & $\mathfrak{s u}(k)$ & $k \geq 4$ \\
3. & $\mathfrak{s u}(2)$ & $\mathfrak{s p}\left(\frac{n-2}{2}\right)$ & $n \geq 2$ \\
4. & $\mathfrak{s u}(3)$ & $\mathfrak{s p}\left(\frac{n-3}{2}\right)$ &
\end{tabular}


Note that each $\mathfrak{l}_{i}$ has a trivial centralizer in $\mathfrak{s u}(k)$ (or in $\mathfrak{s u}(n-k)$ ), so that the centralizer of $\mathfrak{l}$ in $\mathfrak{k}$ coincides with the center $\mathfrak{z}$. Moreover, except in case 1 , when $k=n-k$, we have that $\mathfrak{l}_{1}$ is not isomorphic to $\mathfrak{l}_{2}$, so that $\mathfrak{l} \cong \mathfrak{l}_{1}+\mathfrak{l}_{2}$ or $\mathfrak{l} \cong \mathfrak{l}_{1}+\mathfrak{l}_{2}+\mathfrak{z}$ or $\mathfrak{l} \cong \mathfrak{s u}\left(\frac{n}{2}\right)^{\Delta} \subset \mathfrak{s u}\left(\frac{n}{2}\right)+\mathfrak{s u}\left(\frac{n}{2}\right)+\mathfrak{z}$ or $\mathfrak{l} \cong \mathfrak{s u}\left(\frac{n}{2}\right)^{\Delta}+\mathfrak{z}$. Note also that the scalars are always needed except in case $1, k \neq \frac{n}{2}$, and case $2, k \geq 5$, while the center $\mathfrak{z}$ always acts nontrivially on the tangent space. The case $\mathfrak{l} \cong \mathfrak{z}+\mathfrak{s u}\left(\frac{n}{2}\right)^{\Delta}$ can be excluded simply by the dimensional condition. Summing up we have the following minimal coisotropic actions:

\begin{tabular}{|c|c|c|}
$\mathfrak{l}$ & $M$ & conditions \\
\hline $\mathfrak{s u}(k)+\mathfrak{s u}(n-k)$ & $\operatorname{Gr}(k, n)$ & $2 k \neq n$ \\
$\mathfrak{z}+\mathfrak{s u}(k)+\mathfrak{s u}(k)$ & $\operatorname{Gr}(k, 2 k)$ & \\
$\mathfrak{s p}(2)+\mathfrak{s u}(n-4)$ & $\operatorname{Gr}(4, n)$ & $n \geq 9$ \\
$\mathfrak{z}+\mathfrak{s p}(2)+\mathfrak{s u}(4)$ & $\operatorname{Gr}(4,8)$ & \\
$\mathfrak{z}+\mathfrak{s u}(2)+\mathfrak{s p}(n-1)$ & $\operatorname{Gr}(2,2 n)$ & $n \geq 3$ \\
$\mathfrak{z}+\mathfrak{s u}(3)+\mathfrak{s p}(n-1)$ & $G r(3,2 n+1)$ & $n \geq 3$
\end{tabular}

(2) Suppose $\mathfrak{l}_{i}$ acts irreducibly and $\mathfrak{l}_{j}$ acts reducibly $(i \neq j, 1 \leq i, j \leq 2)$. Assume, for example, $\mathfrak{l}_{1}$ acts irreducibly on $\left(\mathbb{C}^{k}\right)^{*}$ and $\mathfrak{l}_{2}$ reducibly on $\mathbb{C}^{n-k}$. Thus the second factor splits as the direct sum $V_{o} \oplus V^{1} \oplus \cdots \oplus V^{j} \oplus V_{1} \oplus \cdots \oplus V_{r}$ of irreducible $\mathfrak{l}_{2}$-submodules. We denote by $V_{o}$ the submodule on which the action is trivial; while $V^{k}$ are the nontrivial submodules of dimension one and $V_{k}$ the ones that have dimension bigger than 1. Using Theorem 1.3, we have that $\operatorname{dim} V_{o} \leq 2$ and $r+j \leq 2$. We distinguish some cases according to the dimension of $V_{o}$.

When $\operatorname{dim} V_{o}=n-k=2$, we have $r=0$ and $j=0$, recalling that $2 k \leq n$, we conclude that $k=2$. In this case $\mathfrak{l}=\mathfrak{s u}(2)$ because $\mathfrak{l}_{2}=0$; since the scalars cannot be removed when $\mathfrak{s u}(2)$ acts diagonally, the action is not multiplicity free.

If $\operatorname{dim} V_{o}=1$ either $r=1$ and $j=0$ or $r=0$ and $j \leq 1$.

Since the trace $\operatorname{tr}\left(\rho_{2}(X)\right)=0$ for all $X \in \mathfrak{l}$, if $r=0$, then $j=0$ and in particular $k=1(2 k \leq n=k+1)$, contrary to our assumption. We have the following possibilities, from Tables IIa and IIb:

\begin{tabular}{|c|c|c|c|}
$n$. & $\mathfrak{l}_{1}$ & $\mathfrak{l}_{2}$ & conditions \\
\hline 1. & $\mathfrak{s u}(k)$ & $\mathfrak{s u}(n-k-1)$ & $n \geq k+3$ \\
2. & $\mathfrak{s u}(2)$ & $\mathfrak{s p}\left(\frac{n-3}{2}\right)$ & $n \geq 7, k=2$
\end{tabular}

From this we see that $\mathfrak{l} \subseteq \mathfrak{z}+\mathfrak{l}_{1}+\mathfrak{l}_{2}$. Note that when $n>2 k+1$ in case 1 , the scalars can be reduced while in case 2 , scalars can never be reduced. Summing up we conclude that only $\mathfrak{z}+\mathfrak{s u}(k)+\mathfrak{s u}(n-k-1)$ on $G r(k, n), n>2 k+1$, gives rise to a coisotropic action.

The last case arises when $\operatorname{dim} V_{o}=0$. We have $r+j=2$, otherwise we fall again in the case in which both the actions were irreducible.

If $r=2$, by Tables IIa and IIb we have:

\begin{tabular}{|c|c|c|c|}
$n$. & $\mathfrak{l}_{1}$ & $\mathfrak{l}_{2}$ & conditions \\
\hline 1. & $\mathfrak{s u}(2)$ & $\mathfrak{s u}(p)+\mathfrak{s u}(q)$ & $p+q=n-2, \quad p, q \geq 2$ \\
2. & $\mathfrak{s u}(2)$ & $\mathfrak{s u}(p)+\mathfrak{s p}(q)$ & $p+2 q=n-2, \quad p \geq 2, q>1$ \\
3. & $\mathfrak{s u}(2)$ & $\mathfrak{s p}(p)+\mathfrak{s p}(q)$ & $2 p+2 q=n-2, \quad p, q>1$
\end{tabular}

Note that the centralizer is $\mathfrak{z}+\mathbb{R}$, where $\mathbb{R}$ denotes the centralizer of $\mathfrak{l}_{2}$ in $\mathfrak{s u}(n-2)$. We recall briefly the action of $\mathfrak{z}$ and $\mathbb{R}$ on the slice that is given by

$$
\left(\pi^{*} \otimes V_{1}\right) \oplus\left(\pi^{*} \otimes V_{2}\right) .
$$


$\mathfrak{z}$ acts as $\left(e^{i\left(\frac{\phi}{2}+\frac{\phi}{n-2}\right)}, e^{i\left(\frac{\phi}{2}+\frac{\phi}{n-2}\right)}\right)$ while $\mathbb{R}$ acts as $\left(e^{-i \frac{\psi}{p}}, e^{i \frac{\psi}{q}}\right)$.

By Table IIa the scalars, in the first case, can be removed if $p$ and $q$ are bigger than 2 while they cannot be reduced if $p=q=2$. If $p=2$ and $q>2$, the scalars cannot be reduced if the action on the first factor is trivial: this happens when we consider the line $\psi=\frac{n}{n-2} \phi$, which one can show easily that corresponds to the centralizer of $\mathfrak{s u}(n-4)+\mathfrak{s u}(4)$ in $\mathfrak{s u}(n)$.

In the second case the scalars cannot be reduced if $p=2$. If $p>2$, then the scalars are needed if the action on the second factor is trivial, that corresponds to the line $\psi=\frac{-n q}{2(n-2)} \phi$.

Finally, in the third case the scalars can never be reduced.

Here we give the corresponding minimal coisotropic actions. In what follows we will denote by $\mathbb{R}(\alpha)$ the lines different from $y=\alpha x$ where $(x, y) \in \mathfrak{z}+\mathbb{R}$ :

\begin{tabular}{|c|c|c|c|}
$n$. & $\mathfrak{l}$ & $M$ & conditions \\
\hline 1. & $\mathfrak{z}+\mathfrak{s u}(2)+\mathbb{R}+\mathfrak{s u}(2)+\mathfrak{s u}(2)$ & $G r(2,6)$ & \\
2. & $\mathfrak{s u}(2)+\mathfrak{s u}(p)+\mathfrak{s u}(q)$ & $G r(2, n)$ & $p$ and $q>2, p+q=n-2$ \\
3. & $\mathbb{R}\left(\frac{n}{n-2}\right)+\mathfrak{s u}(2)+\mathfrak{s u}(2)+\mathfrak{s u}(n-4)$ & $G r(2, n)$ & $n>6$ \\
4. & $\mathbb{R}\left(\frac{-n q}{2(n-2)}\right)+\mathfrak{s u}(2)+\mathfrak{s u}(p)+\mathfrak{s p}(q)$ & $G r(2, n)$ & $p>2, q>1, n=2+p+2 q$ \\
5. & $\mathfrak{z}+\mathfrak{s u}(2)+\mathbb{R}+\mathfrak{s u}(2)+\mathfrak{s p}(n-2)$ & $G r(2,2 n))$ & $n>3$ \\
6. & $\mathfrak{z}+\mathfrak{s u}(2)+\mathbb{R}+\mathfrak{s p}(p)+\mathfrak{s p}(q)$ & $G r(2,2 n)$ & $p>1, q>1,2 p+2 q=n-2$
\end{tabular}

Suppose now $j=1$ and $r=1$. We obtain

\begin{tabular}{|c|c|c|c|}
$n$. & $\mathfrak{l}_{1}$ & $\mathfrak{l}_{2}$ & conditions \\
\hline 1. & $\mathfrak{s u}(k)$ & $\mathfrak{s u}(n-k-1)+\mathbb{R}$ & $n \geq k+3$ \\
2. & $\mathfrak{s u}(2)$ & $\mathfrak{s p}\left(\frac{n-3}{2}\right)+\mathbb{R}$ & $n \geq 7, k=2$
\end{tabular}

Note that the centralizer is again $\mathfrak{z}+\mathbb{R}$, where $\mathbb{R}$ is the centralizer of $\mathfrak{l}_{2}$ in $\mathfrak{s u}(n-k)$. Clearly $\mathfrak{l}$ can be $\mathfrak{z}+\mathbb{R}+\mathfrak{s u}(k)^{\Delta}$, but this case does not hold for dimensional reasons. The scalars acts as follows: $\mathfrak{z}$ acts as $\left(e^{i\left(\frac{\phi}{k}+\frac{\phi}{n-k}\right)}, e^{i\left(\frac{\phi}{k}+\frac{\phi}{n-k}\right)}\right)$, and $\mathbb{R}$ as $\left(e^{-i \psi}, e^{i \frac{\psi}{n-k-1}}\right)$. Hence, with the same arguments used above, we get:

\begin{tabular}{|c|c|c|c|}
$n$. & $\mathfrak{l}$ & $M$ & conditions \\
\hline 1. & $\mathfrak{z}+\mathfrak{s u}(k)+\mathbb{R}+\mathfrak{s u}(k-1)$ & $G r(k, 2 k)$ & $k \geq 3$ \\
2. & $\mathfrak{z}+\mathfrak{s u}(k)+\mathbb{R}+\mathfrak{s u}(k)$ & $G r(k, 2 k+1)$ & $k \geq 2$ \\
3. & $\mathbb{R}\left(\frac{n}{(n-k) k}\right)+\mathfrak{s u}(k)+\mathfrak{s u}(n-k-1)$ & $G r(k, n)$ & $n>2 k+1$ \\
5. & $\mathfrak{z}+\mathbb{R}+\mathfrak{s u}(2)+\mathfrak{s p}(n-1)$ & $G r(2,2 n+1)$ & $n>2$
\end{tabular}

Finally, if $j=2$, we get that $n-k=2$, hence $k=2, \mathfrak{l}_{2}=\mathfrak{t}_{1} \subseteq \mathfrak{s u}(2)$ is a torus and we have the corresponding diagonal action of $\mathfrak{s u}(2)$ on the slice, given by $\mathbb{C}^{2} \otimes \mathbb{C}^{2}$. Note that, in this case, the centralizer coincides with $\mathfrak{z}+\mathfrak{t}_{1}$, hence the action of $\mathfrak{z}+\mathfrak{t}_{1}+\mathfrak{s u}(2)$ on $\operatorname{Gr}(2,4)$ is multiplicity free but the scalar cannot be reduced.

Now assume $\mathfrak{l}_{1}$ acts reducibly and $\mathfrak{l}_{2}$ acts irreducibly. Following the same procedure used in the symmetric case we find either the dual cases, i.e., where one interchanges the roles of $k$ and $n-k$, or cases already found. Only when, with the same notation used above, $\left(\mathbb{C}^{k}\right)^{*}=W_{o} \oplus W^{1} \oplus \cdots \oplus W^{m} \oplus W_{1} \oplus \cdots \oplus W_{s}$ and $\operatorname{dim} W_{o}=0, m=2$ and $s=0$, we get another different coisotropic action. Clearly, again by Tables IIa and IIb, $k=2$ and on the slice we have $\mathfrak{s u}(n-2)$ or $\mathfrak{s p}\left(\frac{n-2}{2}\right)$ acting diagonally on $\mathbb{C}^{n-2} \otimes \mathbb{C}^{n-2}$. The scalars can be reduced if $n \neq 4$ in the first case, while in the second case the scalars are needed. We note also that $\mathfrak{l}_{1}$ is a torus, denoted by $\mathfrak{t}_{1}$, in $\mathfrak{s u}(2)$. Hence we have, with the same notation used above, 
the following multiplicity free actions:

\begin{tabular}{|c|c|c|c|}
$n$. & $\mathfrak{l}$ & $M$ & conditions \\
\hline 1. & $\mathfrak{t}_{1}+\mathfrak{s u}(n-2)$ & $\operatorname{Gr}(2, n)$ & $n>4$ \\
2. & $\mathfrak{z}+\mathfrak{t}_{1}+\mathfrak{s p}(n-1)$ & $\operatorname{Gr}(2,2 n)$ & $n \geq 2$
\end{tabular}

(3) $\mathfrak{l}_{1}$ and $\mathfrak{l}_{2}$ act both reducibly on $\left(\mathbb{C}^{k}\right)^{*}$ and $\mathbb{C}^{n-k}$ respectively. We can split $\left(\mathbb{C}^{k}\right)^{*}=W_{o} \oplus W^{1} \oplus \cdots \oplus W^{m} \oplus W_{1} \oplus \cdots \oplus W_{s}$ as $\mathfrak{l}_{1}$-irreducible submodules and $\mathbb{C}^{n-k}=V_{o} \oplus V^{1} \oplus \cdots \oplus V^{j} \oplus V_{1} \oplus \cdots \oplus V_{r}$ as $\mathfrak{l}_{2}$-irreducible submodules. First of all note that $r \neq 0$ or $s \neq 0$, otherwise the dimensional condition is not satisfied. Here we investigate the possible dimensions of $V_{o}$ and $W_{o}$. Assume that $V_{o}, W_{o} \neq 0$; then $\mathfrak{l}_{1}+\mathfrak{l}_{2}$ acts trivially on $V_{o} \otimes W_{o}$ and $\mathfrak{l}$ acts coisotropically, so that $\mathfrak{l}$ has no trivial projection along $\mathfrak{z}$ which acts on $V_{o} \otimes W_{o}$. Since $\operatorname{dim} \mathfrak{z}=1$, we must have $\operatorname{dim}\left(V_{o} \otimes W_{o}\right) \leq 1$. On the other hand, $\operatorname{dim} W_{o}, \operatorname{dim} V_{o} \leq 2$. Indeed, assume by contradiction, $\operatorname{dim} W_{o}>2$. Clearly $r=0$, otherwise we have too many terms in the slice; on the other hand, by dimensional reasons, $s$ has to be greater than or equal to 1. Recall also that $\operatorname{tr}\left(\rho_{2}(X)\right)=0, \forall X \in \mathfrak{l}$ so $\operatorname{dim} V_{o}=0$ and $j=2$ or $\operatorname{dim} V_{o}>0$ and $j=0$. The first case is excluded, otherwise $k=2$, which is impossible since $k \geq \operatorname{dim} W_{o}>2$. Hence $\operatorname{dim} V_{o} \neq 0$ so $\operatorname{dim}\left(W_{o} \otimes V_{o}\right) \geq 2$, which is a contradiction.

Note also that we have a symmetry between $W_{o}$ and $V_{o}$ so we have only four cases. Our aim is to prove none of these gives a coisotropic action. We will analyze the one in which $\operatorname{dim} V_{o}=\operatorname{dim} W_{o}=1$ because the others are quite similar. We know that $r$ or $s$ must be positive. Assume, for example, $r \geq 1$. Since $\rho_{1}(l) \subseteq \mathfrak{s u}(k)$, then, again by the condition on the trace, $m=0$. In particular, $s$ has to be greater, as $k>1$, or equal to 1 , so $j=0$ as before. Now in the slice representation appears the indecomposable factor $\left(W_{1} \otimes V_{o}\right) \oplus\left(V_{1} \otimes W_{o}\right) \oplus\left(W_{1} \otimes V_{1}\right)$, hence the action cannot be multiplicity free. Clearly the case $s \geq 1$ is exactly the same.

2.5.2. The case $k<l, k<n-l$. Let $L \subset \mathrm{S}(\mathrm{U}(l) \times \mathrm{U}(n-l))$. Let $\pi_{1}$ be a complex $k$-plane in $\mathbb{C}^{l}$ and let $\pi_{2}$ be a complex $k$-plane in $\mathbb{C}^{n-l}$. Clearly $K \pi_{i}$ are complex orbits; so if $L \subseteq K$ acts on $\operatorname{Gr}(k, n)$ coisotropically, then it acts also on $K \pi_{i}$, $i=1,2$, coisotropically (see 7], Restriction Lemma).

With the same notation used in the previous case we denote by $\mathfrak{l}_{1}$ (resp. $\mathfrak{l}_{2}$ ) the projection of $\mathfrak{l}$ on $\mathfrak{s u}(l)($ resp. $\mathfrak{s u}(n-l))$. We have the following possibilities:

(1) $\mathfrak{l}_{i}, i=1,2$, acts transitively on $K \pi_{i}$;

(2) $\mathfrak{l}_{i}$ acts transitively and $\mathfrak{l}_{j}(\mathfrak{l})$ acts coisotropically $(i \neq j \bmod 2)$;

(3) $\mathfrak{l}_{i}, i=1,2$, acts coistropically.

(1) $\mathfrak{l}_{1}=\mathfrak{s u}(l)$ and $\mathfrak{l}_{2}=\mathfrak{s u}(n-l)$. The simply connected group with Lie algebra $\mathfrak{l}=\mathfrak{l}_{1}+\mathfrak{l}_{2}$ is $L=\mathrm{SU}(l) \times \mathrm{SU}(n-l)$ and $L \pi_{1}$ is a complex orbit. Furthermore, it is easy to see that the slice representation is given by $S=\pi_{1}^{*} \otimes \mathbb{C}^{n-l}$. On the slice $S$ we have, looking at Tables Ia, Ib, the irreducible multiplicity free action of $\mathfrak{s u}(k)+\mathfrak{s u}(n-l)$, where $k \neq n-l$, and $k, n-l \geq 2$. Hence, under these conditions, we get that this action is multiplicity free. Note that when $l=n-l$, then $\mathfrak{l}$ should be also $\mathbb{R}+\mathfrak{s u}(l)^{\Delta}$, but this case is not coisotropic for dimensional reasons.

(2.a) $\mathfrak{l}_{1}=\mathfrak{s u}(l)$ and $\mathfrak{l}_{2}=\mathfrak{s o}(n-l)($ or $\mathfrak{s p}(n-l))$. We can take a complex $\mathfrak{s o}(n-l)$ (resp. $\mathfrak{s p}(n-l)$ )-orbit in $\mathbb{C}^{n-l}$ and it is easy to see that the slice representation contains the submodule $S^{2}\left(\pi_{2}\right) \oplus \pi_{2}^{*} \otimes \mathbb{C}^{l}$ (resp. $\left.\Lambda^{2}\left(\pi_{2}\right) \oplus \pi_{2}^{*} \otimes \mathbb{C}^{l}\right)$. The stabilizer's factor $\mathrm{U}(k)$ acts diagonally on $S^{2}\left(\pi_{2}\right)$ (resp. on $\Lambda^{2}\left(\pi_{2}\right)$ ) and on $\pi_{2}^{*}$. This kind of indecomposable action does not appear in Table II, hence these actions fail to be coisotropic. 
(2.b) $\mathfrak{l}_{1}=\mathfrak{s u}(l)$ and $\mathfrak{l}_{2} \subseteq \mathbb{R}+\mathfrak{s u}(p)+\mathfrak{s u}(q)$, where $p+q=n-l$ and $\mathbb{R}$ is the centralizer of $\mathfrak{l}_{2}$ in $\mathfrak{s u}(n-l)$. The slice representation relative to the complex orbit $\mathrm{SU}(l) \pi_{1}$ is given by $S=\pi_{1}^{*} \otimes \mathbb{C}^{n-l}$. We can decompose $\mathbb{C}^{n-l}$ as $\mathfrak{l}_{2}$-irreducible submodules as $V_{o} \oplus V^{1} \oplus \cdots \oplus V^{j} \oplus V_{1} \oplus \cdots \oplus V_{r}$, with the same notation used in the fixed point case. Note that again $\operatorname{dim} V_{o} \leq 2$ and $r+j \leq 2$. We will follow exactly the same procedure used when $k=l$ distinguishing three different cases, namely when $\operatorname{dim} V_{o}=2,1$ or 0 .

When $\operatorname{dim} V_{o}=2$, we have $\mathbb{C}^{n-l}=V_{o}$ that implies, since $k \neq 1, k=2=n-l$, which is not our case, since $k<n-l$.

If $\operatorname{dim} V_{o}=1$, it is easy to see that $\mathbb{C}^{n-l}$ splits as the direct sum $\mathbb{C} \oplus V_{1}$, i.e., $j=0$ and $r=1$. The slice becomes $S=\pi_{1}{ }^{*} \oplus \pi_{1}{ }^{*} \otimes V_{1}$, hence on the slice $\mathbb{R}_{s}+\mathfrak{s u}(k)+\mathfrak{s u}(n-l-1)$ or $\mathbb{R}_{s}+\mathfrak{s u}(2)+\mathfrak{s p}((n-l-1) / 2)$ act, where $\mathfrak{s u}(k)+\mathbb{R}_{s}$ acts diagonally on $\pi_{1}{ }^{*} \oplus \pi_{1}{ }^{*}$ and where $\mathbb{R}_{s} \subseteq \mathfrak{s u}(l)$ is the determinant condition of the stabilizer's factor $\mathrm{S}(\mathrm{U}(k) \times \mathrm{U}(l-k))$ since $k<l$. The centralizer of $\mathfrak{l}$ coincides with $\mathfrak{c}+\mathbb{R}_{s}$, where the centralizer $\mathfrak{c}$ of $\mathfrak{s u}(l)+\mathfrak{s u}(n-l)$ in $\mathfrak{s u}(n)$ acts as $\left(e^{i\left(\frac{n \phi}{(n-l) l}\right)}, e^{i\left(\frac{n \phi}{(n-l) l}\right)}\right)$, while $\mathbb{R}_{s}$ acts as $\left(e^{i\left(\frac{\psi}{k}\right)}, e^{i\left(\frac{\psi}{k}\right)}\right)$ : that corresponds to a single scalar action. Note that, only in case 1 , when $n>k+l+1$, one can reduce the scalars. Hence we find only the coisotropic action of $\mathfrak{s u}(l)+\mathfrak{s u}(n-l-1)$ on $G r(k, n)$, where $n>k+l+1$.

Suppose now $\operatorname{dim} V_{o}=0$. Assume first $r=2$. The slice $S$ splits as $\pi_{1}{ }^{*} \otimes V_{1} \oplus \pi_{1}{ }^{*} \otimes$ $V_{2}$, hence $k=2$ and on the slice we have the following multiplicity free actions:

\begin{tabular}{|c|c|c|c|}
$n$. & $\mathfrak{h}_{1}$ & $\mathfrak{l}_{2}$ & conditions \\
\hline 1. & $\mathbb{R}_{s}+\mathfrak{s u}(2)$ & $\mathfrak{s u}(p)+\mathfrak{s u}(q)$ & $p+q=n-l, \quad p, q \geq 2$ \\
2. & $\mathbb{R}_{s}+\mathfrak{s u}(2)$ & $\mathfrak{s u}(p)+\mathfrak{s p}(q)$ & $p+2 q=n-l, \quad p \geq 2, q \geq 1$ \\
3. & $\mathbb{R}_{s}+\mathfrak{s u}(2)$ & $\mathfrak{s p}(p)+\mathfrak{s p}(q)$ & $2 p+2 q=n-l, \quad p, q \geq 1$
\end{tabular}

where $\mathfrak{h}_{1}$ denotes the factor of the stabilizer of $\pi_{1}$ that acts on $\pi_{1}{ }^{*}$. Note that the centralizer of $\mathfrak{l}$ in $\mathfrak{k}$ is $\mathfrak{c}+\mathbb{R}_{s}+\mathbb{R}$. Here we give the corresponding minimal coisotropic actions:

\begin{tabular}{|c|c|c|c|}
$n$. & $\mathfrak{l}$ & $M$ & conditions \\
\hline 1. & $\mathfrak{s u}(l)+\mathbb{R}+\mathfrak{s u}(2)+\mathfrak{s u}(2)$ & $G r(2, n)$ & $n=l+4$ \\
2. & $\mathfrak{s u}(l)+\mathfrak{s u}(p)+\mathfrak{s u}(q)$ & $G r(2, n)$ & $p, q>2, p+q=n-l$ \\
3. & $\mathfrak{s u}(l)+\mathfrak{s u}(2)+\mathfrak{s u}(n-l-2)$ & $G r(2, n)$ & $k<l, n-l$ \\
4. & $\mathfrak{s u}(l)+\mathfrak{s u}(p)+\mathfrak{s p}(q)$ & $G r(2, n)$ & $p>2, p+2 q=n-l$ \\
5. & $\mathfrak{s u}(l)+\mathbb{R}+\mathfrak{s u}(2)+\mathfrak{s p}(q)$ & $G r(2, n)$ & $4 \leq 2 q=n-l-2$ \\
6. & $\mathfrak{s u}(l)+\mathbb{R}+\mathfrak{s p}(p)+\mathfrak{s p}(q)$ & $G r(2, n)$ & $2 p+2 q=n-l, p, q \geq 2$
\end{tabular}

Suppose now $j=1$ and $r=1$. We obtain, with the same arguments used in the fixed point case, and recalling that the centralizer is again $\mathfrak{c}+\mathbb{R}$, the following minimal coisotropic actions:

\begin{tabular}{|c|c|c|c|}
$n$. & $\mathfrak{l}$ & $M$ & conditions \\
\hline 1. & $\mathfrak{s u}(l)+\mathbb{R}+\mathfrak{s u}(n-l-1)$ & $G r(k, n)$ & $k=n-l-1 \geq 2$ \\
2. & $\mathfrak{s u}(l)+\mathfrak{s u}(n-l-1)$ & $G r(k, n)$ & $n>k+l+1$ \\
3. & $\mathbb{R}+\mathfrak{s u}(l)+\mathfrak{s p}(n-1)$ & $G r(2,2 n+l-1)$ & $n>2$
\end{tabular}

Finally, if $j=2$ and $r=0$, we get that $n-l=2$, hence $k=1$, which is not our case. 
(3) Note that, by (2.a), we can restrict our analysis to the case in which the two projections are contained into reducible subgroups; that is, $\mathfrak{l}_{1} \subseteq \mathbb{R}+\mathfrak{s u}\left(p_{1}\right)+$ $\mathfrak{s u}\left(q_{1}\right), p_{1}+q_{1}=l$ and $\mathfrak{l}_{2} \subseteq \mathbb{R}+\mathfrak{s u}\left(p_{2}\right)+\mathfrak{s u}\left(q_{2}\right), p_{2}+q_{2}=n-l$. We want to prove that this action fails to be multiplicity free.

Take $\pi_{1}$ a complex $k_{1}$-plane in $\mathbb{C}^{p_{1}}$ and $\pi_{2}$ a complex $k_{2}$-plane in $\mathbb{C}^{q_{1}}$, where $k_{1}+k_{2}=k$. The orbit $\mathrm{S}\left(\mathrm{U}\left(p_{1}\right) \times \mathrm{U}\left(q_{1}\right)\right)\left(\pi_{1} \oplus \pi_{2}\right)$ is complex and it is easy to check that the slice is given by

$$
\left(\pi_{1}^{*} \otimes \pi_{2}^{\perp}\right) \oplus\left(\pi_{1}^{*} \otimes \mathbb{C}^{p_{2}}\right) \oplus\left(\pi_{1}^{*} \otimes \mathbb{C}^{q_{2}}\right) \oplus\left(\pi_{2}^{*} \otimes \pi_{1}^{\perp}\right) \oplus\left(\pi_{2}^{*} \otimes \mathbb{C}^{q_{2}}\right) \oplus\left(\pi_{2}^{*} \otimes \mathbb{C}^{p_{2}}\right),
$$

where $\pi_{1}^{\perp}$ (resp. $\left.\pi_{2}^{\perp}\right)$ denotes the orthogonal complement of $\pi_{1}$ (resp. $\left.\pi_{2}\right)$ in $\mathbb{C}^{p}$ (resp. $\left.\mathbb{C}^{q}\right)$.

Note that only the case $k_{1}=k_{2}=1$ is admissible since, otherwise, there would be at least three terms on which for example $\mathfrak{s u}\left(k_{1}\right)$ (if $k_{1}>1$ ) acts. Then, under this assumption, there must be, for example $p_{1}>1$, otherwise $p_{1}+p_{2}+q_{1}+q_{2}=n=4$, and the corresponding action is not coisotropic for dimensional reasons. Take a 2 -plane $\pi$ in $\mathbb{C}^{p_{1}}$, then in the slice appears the term $\pi^{*} \otimes \mathbb{C}^{p_{2}} \oplus \pi^{*} \otimes \mathbb{C}^{q_{1}} \oplus \pi^{*} \otimes \mathbb{C}^{q_{2}}$, hence the action is not coisotropic.

2.5.3. The case $k<l, k>n-l$. We can take a $k$-plane $\pi \subseteq \mathbb{C}^{l}$ and determine the complex $K$-orbit. By the restriction lemma we have that the projection $\rho_{1}(\mathfrak{l})=\mathfrak{l}_{1}$ must act coisotropically on the complex orbit $G r(k, l)$. Therefore we may have two possible situations, namely when the action is transitive, i.e., $\mathfrak{l}_{1}=\mathfrak{s u}(l)$, or when $\mathfrak{l}_{1}$ acts coisotropically on $\operatorname{Gr}(k, l)$.

(1) Suppose $\mathfrak{l}_{1}=\mathfrak{s u}(l)$. Note that $\mathfrak{l}_{1}$ acts on the first factor of the slice $S=\pi^{*} \otimes$ $\mathbb{C}^{n-l}$ irreducibly; while the second factor can split as the direct sum of irreducible $\mathfrak{l}_{2}$-submodules. Following the same procedure we have used when both $l, n-l>k$, and with the same notation, we have $\operatorname{dim} V_{o} \leq 2$, and $\mathbb{C}^{n-l}$ can split as at most two summands.

When $\operatorname{dim} V_{o}=2$, the action is not coisotropic, as the diagonal action of $\mathfrak{s u}(k)$ needs the scalars indeed the center acts as $z \rightarrow\left(z^{a}, z^{a}\right)$.

If $\operatorname{dim} V_{o}=1$, then $r=1$ and $j=0$, or $j=r=0$; in the first case the only possibility is the action of $\mathfrak{s u}(k)+\mathfrak{s u}(n-l-1), n-l \geq 3$, on the slice; note that the scalars are removable if and only if $n-l-1>k$ which is not our case, hence the action is not multiplicity free. In the second case, by Table Ia, we get the multiplicity free action of $\mathfrak{s u}(n-1)$.

Finally, if $\operatorname{dim} V_{o}=0$, we obtain the same cases found in (2.b) (when $\operatorname{dim} V_{o}=$ $0)$. However, note that the groups arising when $r=2$ and $j=0$ have to be excluded since, otherwise, by Tables IIa, IIb, $k=2$ and $2>n-l=\operatorname{dim} V_{1}+\operatorname{dim} V_{2} \geq 4$ that gives rise to a contradiction. On the other hand, the case arising when $j=2$ and $r=0$ has to be considered. In this situation $\mathfrak{l}_{2}=\mathfrak{t}_{1}$ and $l=n-2$.

Summing up, when the $\mathfrak{l}_{2}$-action is reducible, we get the following coisotropic actions:

\begin{tabular}{|c|c|c|c|}
$n$. & $\mathfrak{l}$ & $M$ & conditions \\
\hline 1. & $\mathfrak{s u}(l)+\mathbb{R}+\mathfrak{s u}(n-l-1)$ & $G r(k, n)$ & $n \geq l+5, k>n-l$ \\
2. & $\mathfrak{t}_{1}+\mathfrak{s u}(n-2)$ & $G r(k, n)$ & $k>2$
\end{tabular}


When the $\mathfrak{l}_{2}$-action is irreducible, with the same arguments that we have used in the fixed point case, we have the following multiplicity free actions:

\begin{tabular}{|c|c|c|c|}
$n$ & $\mathfrak{l}$ & $M$ & conditions \\
\hline 1. & $\mathfrak{s u}(n-1)$ & $G r(k, n)$ & $n \geq 4$ \\
2. & $\mathfrak{s u}(l)+\mathfrak{s u}(n-l)$ & $G r(k, n)$ & $k, n-l \geq 2$ \\
3. & $\mathfrak{s p}(2)+\mathfrak{s u}(n-4)$ & $G r(k, n)$ & $5<k \leq \frac{n}{2}$
\end{tabular}

(2) Suppose now that $\mathfrak{l}_{1}$ acts coisotropically on $\operatorname{Gr}(k, l)$. We will test all the maximal subgroups of $\mathrm{SU}(l)$ that act multiplicity free on $\operatorname{Gr}(k, l)$ and then, according to the analysis that we have already done, we will go through all their subgroups still acting coisotropically. The idea will be always the same, we choose a complex orbit, we find the slice representation and we determine under which conditions the action appears in Tables Ia or IIa, IIb.

$\mathfrak{l}_{1}=\mathfrak{s o}(l)$. The slice $S=S^{2}\left(\pi^{*}\right) \oplus\left(\pi^{*} \otimes \mathbb{C}^{n-l}\right)$ does not appear in Tables IIa, IIb; hence the action is not coisotropic.

$\mathfrak{l}_{1}=\mathfrak{s p}(l / 2)$. The slice is $S=\Lambda^{2}\left(\pi^{*}\right) \oplus\left(\pi^{*} \otimes \mathbb{C}^{n-l}\right)$ where $\mathrm{U}(k)$ acts diagonally on $\Lambda^{2}\left(\pi^{*}\right) \oplus \pi^{*}$. It appears in Table IIa or IIb iff $n-l=1$, i.e., $l=n-1$. The scalars act on the slice as $z \mapsto\left(z^{a}, z^{a}\right)$. Therefore, by Tables IIa and IIb, $\mathfrak{s p}((n-1) / 2) \subseteq \mathfrak{s u}(n-1)$ acts coisotropically on $G r(k, n)$ for $k \neq 3$; if $k=3$, the slice becomes $S=\pi^{*} \oplus \pi^{*}$ and $\mathbb{R}+\mathfrak{s p}(n)$ act coisotropically on $G r(3,2 n+1)$. Note that no subgroup of $\operatorname{Sp}(n-1 / 2)$ can act coisotropically on the complex Grassmannian.

$\mathfrak{l}_{1}=\mathbb{R}+\mathfrak{s u}(p)+\mathfrak{s u}(q)$ where $p+q=l$ and $\mathbb{R}$ is the centralizer of $\mathfrak{s u}(p)+\mathfrak{s u}(q)$ in $\mathfrak{s u}(l)$. We take $k_{1}, k_{2}$ such that $k_{1} \leq p, k_{2} \leq q$ and $k=k_{1}+k_{2}$. Let $\pi_{1}$ and $\pi_{2}$ be $k_{1}$ - and $k_{2}$-planes in $\mathbb{C}^{p}$ and $\mathbb{C}^{q}$ respectively; let $\pi=\pi_{1} \oplus \pi_{2}$ be the $k$-complex plane in $\mathbb{C}^{l}$. The slice is

$$
S=\left(\pi_{1}{ }^{*} \otimes \mathbb{C}^{n-l}\right) \oplus\left(\pi_{2}{ }^{*} \otimes \mathbb{C}^{n-l}\right) \oplus\left(\pi_{1}{ }^{*} \otimes \pi_{2}^{\perp}\right) \oplus\left(\pi_{2}{ }^{*} \otimes \pi_{1}^{\perp}\right) .
$$

We are going to prove that, for all $k, n-l$ has to be 1 and $\mathfrak{l}_{2}=0$. Indeed, if $k=2$ and since $k>n-l$, then $n-l=1$. For $k \geq 3$, the group acts on the slice coisotropically only if $n-l=1$, i.e., $p+q=n-1$, note that if it is not the case, there would be too many terms.

By a straightforward calculation we can always take $p$ or $q$ bigger than $k$, otherwise we fall again in the fixed point case. Assume, for example, $p>k$ and take a complex $k$-plane $\pi \subset \mathbb{C}^{p}$ and consider the complex orbit through $\pi$. The slice representation is given by $\left(\pi^{*} \otimes \mathbb{C}^{q}\right) \oplus\left(\pi^{*} \otimes \mathbb{C}\right)$ where $\mathbb{R}_{s}+\mathfrak{s u}(k)$ acts diagonally on $\pi^{*}$; here $\mathbb{R}_{s}$ denotes the centralizer of $\mathfrak{s u}(k)+\mathfrak{s u}(p-k)$ in $\mathfrak{s u}(p)$.

The centralizer, $\mathfrak{c}$, of $\mathfrak{s u}(n-1)$ in $\mathfrak{s u}(n)$, acts on the slice as $\left(1, e^{-i \frac{n \phi}{n-1}}\right)$; while $\mathbb{R}$ as $\left(e^{-i \frac{\psi}{p}} e^{-i \frac{\psi}{q}}, e^{-i \frac{\psi}{p}}\right)$ and $\mathbb{R}_{s}$ as $\left(e^{i \frac{\theta}{k}}, e^{i \frac{\theta}{k}}\right)$.

If $q=1$, we shall assume $k \geq 3$ since if it is not, we fall again in the fixed point case. The slice is given by $\pi^{*} \oplus \pi^{*}$ and the scalars act on the slice $\left(e^{-i \frac{\psi}{p}} e^{-i \psi} e^{i \frac{\theta}{k}}\right.$, $\left.e^{-i \frac{n \phi}{n-1}} e^{-i \frac{\psi}{p}} e^{i \frac{\theta}{k}}\right)$. We know that this action fails to be multiplicity free when $a=b$. We conclude, when $q=1$, that the action is coisotropic if and only if $\psi \neq \frac{n \phi}{n-1}$. Note also that the line $\psi=\frac{n \psi}{n-1}$ corresponds to the centralizer of $\mathfrak{s u}(2)+\mathfrak{s u}(n-2)$ in $\mathfrak{s u}(n)$. In particular, if $\mathfrak{t}_{1}$ denotes a torus in $\mathfrak{s u}(2)$, then the action is multiplicity free if and only if the projection of the scalar has a no trivial component along $\mathfrak{t}_{1}$.

Now, suppose that $q>1$. The slice becomes $\left(\pi^{*} \otimes \mathbb{C}^{q}\right) \oplus\left(\pi^{*} \otimes \mathbb{C}\right)$ and the action of the scalars on the slice is $\left(e^{-i \frac{\psi}{p}} e^{-i \frac{\psi}{q}} e^{i \frac{\theta}{k}}, e^{-i \frac{n \phi}{n-1}} e^{-i \frac{\psi}{p}} e^{i \frac{\theta}{k}}\right)$. 
By Tables IIa, IIb we can distinguish some cases according to the relations between $k$ and $q$.

First, if $q>k$, one can reduce the scalars if and only if the action on the second factor is nontrivial and this holds since $\mathbb{R}_{s}$ acts.

When $q=k$, we fall again in the fixed point case.

In case $q=k-1$ the scalars cannot be reduced and it is easy to check that the image of the scalars's action is one dimensional if and only if $\psi=\frac{(k-1) n \phi}{n-1}$.

Finally, if $q<k-1$, the action fails to be multiplicity free if and only if the image of the scalars is one dimensional and on both factors the action is the same. This happens on the line $\psi=\frac{q n \phi}{n-1}$. Summing up we have the following minimal coisotropic actions:

\begin{tabular}{|c|c|c|c|}
$n$. & $\mathfrak{l}$ & $M$ & conditions \\
\hline 1. & $\mathfrak{t}_{1}+\mathfrak{s u}(n-2)$ & $G r(k, n)$ & $k>2$ \\
2. & $\mathfrak{s u}(p)+\mathfrak{s u}(q)$ & $G r(k, n)$ & $p+q=n-1, p, q>k$ \\
3. & $\mathbb{R}\left(\frac{(k-1) n}{n-1}\right)+\mathfrak{s u}(k-1)+\mathfrak{s u}(n-k)$ & $G r(k, n)$ & $k \geq 3$ \\
4. & $\mathbb{R}\left(\frac{q n}{n-1}\right)+\mathfrak{s u}(p)+\mathfrak{s u}(q)$ & $G r(k, n)$ & $p+q=n-1, p>k, k \geq q+2$.
\end{tabular}

We shall investigate if we can go under $\mathbb{R}+\mathfrak{s u}(p)+\mathfrak{s u}(q)$.

We first consider the maximal subalgebras of $\mathfrak{s u}(q)$. Choose a $k$-plane $\pi$ in $\mathbb{C}^{p}$ as above. The slice, corresponding to the orbit through $\pi$ is given by $S=$ $\pi^{*} \otimes \mathbb{C}^{q} \oplus \pi^{*} \otimes \mathbb{C}$. By Tables IIa and IIb we argue that no subalgebras give rise to a coisotropic action.

Now consider the maximal subalgebras of $\mathfrak{s u}(p)$. We take the subalgebra $\mathfrak{s o}(p)$ (resp. $\mathfrak{s p}(p / 2))$. Take the $k$-plane $\pi$ such that the orbit through it is complex. The slice becomes $S=S^{2}\left(\pi^{*}\right) \oplus\left(\pi^{*} \otimes \mathbb{C}\right) \oplus\left(\pi^{*} \otimes \mathbb{C}^{q}\right)$ (resp. $S=\Lambda^{2}\left(\pi^{*}\right) \oplus\left(\pi^{*} \otimes \mathbb{C}\right) \oplus$ $\left.\left(\pi^{*} \otimes \mathbb{C}^{q}\right)\right)$ and the corresponding actions on it do not appear in Tables IIa and IIb. The case of a simple subalgebra can be treated likewise.

We now investigate $\mathbb{R}+\mathfrak{s u}\left(p_{1}\right)+\mathfrak{s u}\left(p_{2}\right) \subset \mathfrak{s u}(p)$. We take $k_{1}, k_{2}$ such that $k_{1} \leq p_{1}, k_{2} \leq p_{2}$ and $k=k_{1}+k_{2}$. Let $\pi_{1}$ and $\pi_{2}$ be $k_{1}$ - and $k_{2}$-planes in $\mathbb{C}^{p_{1}}$ and $\mathbb{C}^{p_{2}}$ respectively; let $\pi=\pi_{1} \oplus \pi_{2}$ be the $k$-complex plane in $\mathbb{C}^{p}$. The slice contains the submodules $\left(\pi_{i}{ }^{*} \otimes \mathbb{C}\right) \oplus\left(\pi_{i}{ }^{*} \otimes \mathbb{C}^{q}\right) \oplus\left(\pi_{i}^{*} \otimes \pi_{j}^{+}\right)$with $i \neq j$, hence the corresponding action is not coisotropic.

Finally, the case $\mathfrak{l}_{1}=\mathbb{R}+\mathbb{R}+\mathfrak{s u}(p)^{\Delta}$ can be excluded for dimensional reasons.

\section{Polar actions on COMplex Grassmannians}

In this section we study which coisotropic actions are polar. First observe that the reducible actions arising from Table IIa and IIb are not polar; this can be easily deduced as an application of Theorem 2 (p. 313) [2]; while (see [5] and [9]) in the irreducible case we know that $\mathfrak{s o}(n)$ on $\operatorname{Gr}(k, n), \mathfrak{s p}(n)$ on $\operatorname{Gr}(k, 2 n)$ and $\mathfrak{s u}(l)+\mathfrak{s u}(n-l)$, for $l \neq n-l$ on $G r(k, n)$ give rise to polar actions. The last two cases $\mathfrak{s p i n}(7)$ on $\operatorname{Gr}(2,8)$ and $\mathfrak{s p}(n)$ on $G r(k, 2 n+1)$ can be excluded. The first one fails to be polar since $\mathfrak{s p i n}(7)$ has the same orbits of $\mathfrak{u}(3)$ which is not polar for [5].

We now consider the action of $\mathfrak{s p}(n)$ on $G r(k, 2 n+1)$. For $k \geq 3$, using Theorem 2 in [2], one can easily prove that the action is not polar. Note that if $k=4$, then the action on $S=\Lambda^{2}\left(\pi^{*}\right) \oplus \pi^{*}$ appears in [2] but as a real representation.

Now we consider the case $k=2$. As $\mathfrak{s p}(n)$-module $\mathbb{C}^{2 n+1}=\mathbb{C} \oplus \mathbb{C}^{2 n}$ and take $\pi$ in $\mathbb{C}^{2 n}$ such that the orbit through $\pi$ is complex. The normal space $N=\Lambda^{2}\left(\pi^{*}\right) \oplus \mathbb{C}^{2}$ is acted on by $\operatorname{Sp}(n)_{\pi}=\mathrm{U}(2) \times \operatorname{Sp}(n-2)$, so that the action has cohomogeneity 
2. If the $\operatorname{Sp}(n)$ action were polar, using Proposition 1 in [2] a section in $N$ can be taken as the direct sum of a section for the action of $\mathrm{U}(2)$ on $\Lambda^{2}\left(\pi^{*}\right)$ plus a section of $\mathrm{U}(2)$ on $\mathbb{C}^{2}$. With this remark any subspace $\mathfrak{m} \subseteq N$ generated by $v_{1} \in \Lambda^{2}\left(\pi^{*}\right)$ and $v_{2}$ in $T_{\pi} G r(2,2 n)^{\perp} \subseteq T_{\pi} G r(2,2 n+1)$ would be the tangent space to a totally geodesic submanifold. A direct inspection taking

$$
v_{1}=\left(\begin{array}{cccccc}
0 & 0 & 0 & 1 & \ldots & 0 \\
0 & 0 & -1 & 0 & \ldots & \vdots \\
0 & 1 & 0 & 0 & \ldots & \vdots \\
-1 & 0 & 0 & 0 & \ldots & \vdots \\
\ldots & \ldots & \ldots & \ldots & \ldots & \vdots \\
0 & \ldots & \ldots & \ldots & \ldots & 0
\end{array}\right), v_{2}=\left(\begin{array}{ccccc|c}
0 & 0 & \ldots & 0 & \ldots & 1 \\
0 & 0 & \ldots & 0 & \ldots & 0 \\
\ldots & \ldots & \ldots & 0 & \ldots & \vdots \\
0 & 0 & 0 & 0 & \ldots & \vdots \\
\ldots & \ldots & \ldots & \ldots & \ldots & 0 \\
\hline-1 & 0 & \ldots & \ldots & 0 & 0
\end{array}\right)
$$

shows, together with Theorem 7.2 , p. 224, in 6] on Lie triple systems, that the section $\Sigma=\exp (\mathfrak{m})$ is not totally geodesic, hence the action cannot be polar.

Finally, observe that the groups $K$ that are listed in Table 3 act also hyperpolarly on complex Grassmannians, since $K$ and $\mathrm{S}(\mathrm{U}(l) \times \mathrm{U}(n-l))$ are symmetric subgroups of $\mathrm{SU}(n)$.

\section{Appendix}

In Tables Ia, Ib, IIa and IIb we use the notation of [1]; as an example $\mathfrak{s u}(n) \oplus_{\mathfrak{s u}(n)}$ $\mathfrak{s u}(n)$ denotes the Lie algebra $\mathfrak{s u}(n)$ acting on $\mathbb{C}^{n} \oplus \mathbb{C}^{n}$ via the direct sum of two copies of the natural representation.

In Table $\mathrm{V} \rho_{1}$ and $\Lambda_{i}$ denote the standard representation of GL(1) and the fundamental highest weights respectively.

TABLE Ia. Lie algebras $\mathfrak{k}$ s.t. $\mathbb{R}+\mathfrak{k}$ gives rise to irreducible multiplicity free actions

\begin{tabular}{|lllr|}
\hline $\mathfrak{s u}(n)$ & $n \geq 1$ & $\mathfrak{s o}(n)$ & $n \geq 3$ \\
$\mathfrak{s p}(n)$ & $n \geq 2$ & $S^{2}(\mathfrak{s u}(n))$ & $n \geq 2$ \\
$\Lambda^{2}(\mathfrak{s u}(n))$ & $n \geq 4$ & $\mathfrak{s u}(n) \otimes \mathfrak{s u}(m)$ & $n, m \geq 2$ \\
$\mathfrak{s u}(2) \otimes \mathfrak{s p}(n)$ & $n \geq 2$ & $\mathfrak{s u}(3) \otimes \mathfrak{s p}(n)$ & $n \geq 2$ \\
$\mathfrak{s u}(n) \otimes \mathfrak{s p}(2)$ & $n \geq 4$ & $\mathfrak{s p i n}(7)$ & \\
$\mathfrak{s p i n}(9)$ & & $\mathfrak{s p i n}(10)$ & \\
$\mathfrak{g}_{2}$ & $n \geq 1$ & $\mathfrak{e}_{6}$ & $n \geq 3$ \\
\hline
\end{tabular}

TABLE Ib. Irreducible coisotropic actions in which the scalars are removable

\begin{tabular}{|lllr|}
\hline $\mathfrak{s u}(n)$ & $n \geq 2$ & $\mathfrak{s p}(n)$ & $n \geq 2$ \\
$\Lambda^{2}(\mathfrak{s u}(n))$ & $n \geq 4$ & $\mathfrak{s u}(n) \otimes \mathfrak{s u}(m)$ & $n, m \geq 2, n \neq m$ \\
$\mathfrak{s p i n}(10)$ & & $\mathfrak{s u}(n) \otimes \mathfrak{s p}(2)$ & $n \geq 5$ \\
\hline
\end{tabular}


TABLE IIa. Indecomposable coisotropic actions in which the scalars can be removed or reduced

\begin{tabular}{|c|c|}
\hline 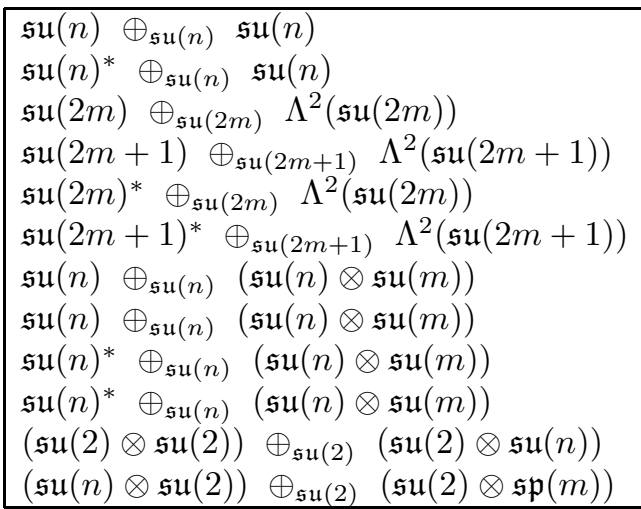 & $\begin{array}{r}n \geq 3, a \neq b \\
n \geq 3 a \neq-b \\
m \geq 2, b \neq 0 \\
\geq 2, a \neq-m b \\
m \geq 2, b \neq 0 \\
m \geq 2, a \neq m b \\
\leq n<m, a \neq 0 \\
\geq m+2, a \neq b \\
\leq n<m, a \neq 0 \\
\geq m+2, a \neq b \\
n \geq 3, a \neq 0 \\
3, m \geq 4, b \neq 0 \\
\end{array}$ \\
\hline
\end{tabular}

TABLE IIb. Indecomposable coisotropic actions in which the scalars cannot be removed or reduced

\begin{tabular}{|lrr|}
\hline $\mathfrak{s u}(2) \oplus_{\mathfrak{s u}(2)}$ & $\mathfrak{s u}(2)$ & \\
$\mathfrak{s u}(n)^{(*)} \oplus_{\mathfrak{s u}(n)^{*}(\mathfrak{s u}(n) \oplus \mathfrak{s u}(n))}$ & $n \geq 2$ \\
$\left(\mathfrak{s u}(n+1)^{(*)} \oplus_{\mathfrak{s u}(n+1)}(\mathfrak{s u}(n+1) \otimes \mathfrak{s u}(n))\right.$ & $n \geq 2$ \\
$\left(\mathfrak{s u}(2) \oplus_{\mathfrak{s u}(2)} \quad(\mathfrak{s u}(2) \otimes \mathfrak{s p}(m))\right.$ & $m \geq 2$ \\
$\left(\mathfrak{s u}(2) \oplus_{\mathfrak{s u}(2))} \oplus_{\mathfrak{s u}(2)}(\mathfrak{s u}(2) \otimes \mathfrak{s p}(m))\right.$ & \\
$\left(\mathfrak{s p}(n) \oplus_{\mathfrak{s u}(2))} \oplus_{\mathfrak{s u}(2)}(\mathfrak{s u}(2) \otimes \mathfrak{s p}(m))\right.$ & $n, m \geq 2$ \\
$\mathfrak{s p}(n) \oplus_{\mathfrak{s p}(n)} \mathfrak{s p}(n)$ & & $n \geq 2$ \\
$\mathfrak{s p i n}(8) \oplus_{\mathfrak{s p}(8)} \quad \mathfrak{s o}(8)$ & \\
\hline
\end{tabular}

TABLE III. Maximal subgroups of $\mathrm{SU}(n)$

\begin{tabular}{|r|c|l|}
\hline$i)$ & $\mathrm{SO}(n)$ & \\
\hline$i i)$ & $\mathrm{Sp}(m)$ & $2 m=n$ \\
\hline$i i i)$ & $\mathrm{S}(\mathrm{U}(k) \times \mathrm{U}(n-k))$ & $1 \leq k \leq n-1$ \\
\hline$i v)$ & $\mathrm{SU}(p) \otimes \mathrm{SU}(q)$ & $p q=n, p \geq 3, q \geq 3$ \\
\hline$v)$ & $\rho(H)$ & $H$ simple $\rho \in \operatorname{Irr}_{\mathbb{C}}, \operatorname{deg} \rho=\mathrm{n}$ \\
\hline
\end{tabular}

TABLE IV. Maximal subgroups of $\operatorname{Sp}(n)$

\begin{tabular}{|r|c|l|}
\hline$i)$ & $\mathrm{U}(n)$ & \\
\hline$i i)$ & $\mathrm{Sp}(k) \times S p(n-k)$ & $1 \leq k \leq n-1$ \\
\hline$i i i)$ & $\mathrm{SO}(p) \otimes \operatorname{Sp}(q)$ & $p q=n, p \geq 3, q \geq 1$ \\
\hline$i v)$ & $\rho(H)$ & $H$ simple $\rho \in \operatorname{Irr}_{\mathbb{H}}, \operatorname{deg} \rho=2 \mathrm{n}$ \\
\hline
\end{tabular}


TABLE V. Reduced prehomogeneous triplets

\begin{tabular}{|c|c|c|c|}
\hline$G$ & $\rho$ & $V$ & conditions \\
\hline $\operatorname{GL}(n)$ & $2 \Lambda_{1}$ & $V\left(\frac{n(n+1)}{2}\right)$ & $n \geq 2$ \\
\hline $\operatorname{GL}(2 n)$ & $\Lambda_{2}$ & $V(n(n-3))$ & $n \geq 3$ \\
\hline $\mathrm{GL}(2)$ & $3 \Lambda_{1}$ & $V(4)$ & \\
\hline $\mathrm{GL}(6)$ & $\Lambda_{3}$ & $V(20)$ & \\
\hline $\operatorname{GL}(7)$ & $\Lambda_{3}$ & $V(35)$ & \\
\hline $\mathrm{GL}(8)$ & $\Lambda_{3}$ & $V(56)$ & \\
\hline $\mathrm{SL}(3) \times \mathrm{GL}(2)$ & $2 \Lambda_{1} \otimes \Lambda_{1}$ & $V(6) \otimes V(2)$ & \\
\hline $\mathrm{SL}(6) \times \mathrm{GL}(2)$ & $\Lambda_{2} \otimes \Lambda_{1}$ & $V(15) \otimes V(2)$ & \\
\hline $\mathrm{SL}(5) \times \mathrm{GL}(3)$ & $\Lambda_{2} \otimes \Lambda_{1}$ & $V(15) \otimes V(3)$ & \\
\hline $\mathrm{SL}(5) \times \mathrm{GL}(4)$ & $\Lambda_{2} \otimes \Lambda_{1}$ & $V(10) \otimes V(4)$ & \\
\hline $\mathrm{SL}(3) \times \mathrm{SL}(3) \times \mathrm{GL}(2)$ & $\Lambda_{1} \otimes \Lambda_{1} \otimes \Lambda_{1}$ & $V(3) \otimes V(3) \otimes V(2)$ & \\
\hline $\operatorname{Sp}(n) \times \operatorname{GL}(2 m)$ & $\Lambda_{1} \otimes \Lambda_{1}$ & $V(2 n) \otimes V(2 m)$ & $n \geq 2 m \geq 2$ \\
\hline $\mathrm{GL}(1) \times \mathrm{Sp}(3)$ & $\rho_{1} \otimes \Lambda_{3}$ & $V(1) \otimes V(14)$ & \\
\hline $\mathrm{SO}(n) \times \mathrm{GL}(m)$ & $\Lambda_{1} \otimes \Lambda_{1}$ & $V(n) \otimes V(m)$ & $n \geq 3, \frac{n}{2} \geq m \geq 1$ \\
\hline $\mathrm{GL}(1) \times \operatorname{Spin}(7)$ & $\rho_{1} \otimes$ spin rep. & $V(1) \otimes V(8)$ & \\
\hline $\operatorname{Spin}(7) \times \operatorname{GL}(2)$ & spin rep $\otimes \Lambda_{1}$ & $V(8) \otimes V(2)$ & \\
\hline $\operatorname{Spin}(7) \times \operatorname{GL}(3)$ & spin rep $\otimes \Lambda_{1}$ & $V(8) \otimes V(3)$ & \\
\hline $\operatorname{GL}(1) \times \operatorname{Spin}(9)$ & $\rho_{1} \otimes$ spin rep. & $V(1) \otimes V(16)$ & \\
\hline $\operatorname{Spin}(10) \times \operatorname{GL}(2)$ & half - spin rep. $\otimes \Lambda_{1}$ & $V(16) \otimes V(2)$ & \\
\hline $\operatorname{Spin}(10) \times \operatorname{GL}(3)$ & half - spin rep. $\otimes \Lambda_{1}$ & $V(16) \otimes V(3)$ & \\
\hline $\mathrm{GL}(1) \times \operatorname{Spin}(11)$ & $\rho_{1} \otimes$ spin. rep. & $V(1) \otimes V(32)$ & \\
\hline $\operatorname{GL}(1) \times \operatorname{Spin}(11)$ & $\rho_{1} \otimes$ half - spin rep. & $V(1) \otimes V(64)$ & \\
\hline $\mathrm{GL}(1) \times \mathrm{G}_{2}$ & $\rho_{1} \otimes \Lambda_{2}$ & $V(1) \otimes V(7)$ & \\
\hline $\mathrm{G}_{2} \times \mathrm{GL}(2)$ & $\Lambda_{2} \otimes \Lambda_{1}$ & $V(7) \otimes V(2)$ & \\
\hline $\mathrm{GL}(1) \times \mathrm{E}_{6}$ & $\rho_{1} \otimes \Lambda_{1}$ & $V(1) \otimes V(27)$ & \\
\hline $\mathrm{E}_{6} \times \mathrm{GL}(2)$ & $\Lambda_{1} \otimes \Lambda_{1}$ & $V(27) \otimes V(2)$ & \\
\hline $\mathrm{GL}(1) \times \mathrm{E}_{7}$ & $\rho_{1} \otimes \Lambda_{1}$ & $V(1) \otimes V(56)$ & \\
\hline $\operatorname{Sp}(n) \times \operatorname{GL}(2)$ & $\Lambda_{1} \otimes 2 \Lambda_{1}$ & $V(2 n) \otimes V(3)$ & \\
\hline $\mathrm{GL}(1) \times \mathrm{Sp}(n) \times \mathrm{SO}(3)$ & $\rho_{1} \otimes \Lambda_{1} \otimes \Lambda_{1}$ & $V(1) \otimes V(2 n) \otimes V(3)$ & \\
\hline $\mathrm{SL}(n) \times \mathrm{GL}(m)$ & $\Lambda_{1} \otimes \Lambda_{1}$ & $V(n) \otimes V(m)$ & $\frac{m}{2} \geq n \geq 1$ \\
\hline $\mathrm{GL}(2 m+1)$ & $\Lambda_{2}$ & $V(m(2 m+1))$ & $m \geq 2$ \\
\hline $\mathrm{SL}(2 m+1) \times \mathrm{GL}(2)$ & $\Lambda_{2} \otimes \Lambda_{1}$ & $V(m(2 m+1)) \otimes V(2)$ & $m \geq 2$ \\
\hline $\operatorname{Sp}(n) \times \operatorname{GL}(2 m+1))$ & $\Lambda_{1} \otimes \Lambda_{1}$ & $V(2 n) \otimes V(2 m+1)$ & $n>2 m+1 \geq 1$ \\
\hline $\mathrm{GL}(1) \times \operatorname{Spin}(10)$ & $\rho_{1} \otimes$ half - spin rep. & $V(1) \otimes V(16)$ & \\
\hline
\end{tabular}

\section{REFERENCES}

[1] Benson, C., Ratcliff, G.: A classification of multiplicity free actions, J. Algebra, 181, 152-186 (1996). MR:1382030 (97c:14046)

[2] Bergmann I.: Reducible polar representations, Manus. Math., 104, 309-324 (2001). MR.1828877 (2003d:53082)

[3] Dadok J.: Polar cordinates induced by actions of compact Lie Groups, Trans. Am. Math. Soc., 288, 125-137 (1985). MR0773051 (86k:22019)

[4] Guillemin, V. Sternberg, S.: Symplectic Techniques in Physics Cambridge: Cambridge University Press, 1984. MR0770935 (86f:58054)

[5] Heintze, E. Eschenburg J.: On the classification of Polar representation, Math. Z., 286, 391-398 (1999). MR1719714 (2001g:53099)

[6] Helgason, S.: Differential Geometry, Lie Groups and Symmetric Spaces, New York-London: Academic Press, 1978. MR0514561 (80k:53081)

[7] Huckleberry, A.T., WurzBacher, T.: Multiplicity-free complex manifolds, Math. Annalen., 286, 261-280 (1990). MR1032934 (91c:32027)

[8] KAC, V.G.: Some remarks on Nilpotent orbit, J. Algebra, 64, 190-213 (1980). MR0575790 (81i:17005) 
[9] Kollross, A.: A classification of hyperpolar and cohomogeneity one actions, Trans. Am. Math. Soc., 354, 571-612 (2002). MR1862559 (2002g:53091)

[10] Kraft, H.: Geometrische Methoden in der Invariantentheorie, Braunschweig, Wiesbaden: Vieweg 1984. MR0768181 (86j:14006)

[11] ONISHCHIK, A. L.: Inclusion relations among transitive compact transformation groups, Trans. Am. Math. Soc., 50, 5-58 (1966).

[12] Podestà, F., Thorbergsson, G.: Polar and Coisotropic Actions on Kähler Manifolds, Trans. Am. Math. Soc., 354, 236-238 (2002). MR.1881015(2002j:53096)

[13] Podestà, F., Thorbergsson, G.: Polar Actions on rank one Symmetric Spaces, J. of Differential Geom., 53, 131-175 (1999). MR1776093 (2001j:53064)

[14] Sato, M., Kimura, T.: A classification of irreducible prehomogeneous vector spaces and their relative invariants, Nagoya Math. J., 65, 1-155 (1977). MR0430336 (55:3341)

Dipartimento di Matematica e Applicazioni per l'Architettura, Università di Firenze, Piazza Ghiberti 27, 50142 Firenze, Italy

E-mail address: biliotti@math.unifi.it

Dipartimento di Matematica e Applicazioni per l'Architettura, Università di Firenze, Piazza Ghiberti 27, 50142 Firenze, Italy

E-mail address: gori@math.unifi.it 